INTER NATIONAL MONETARY FUND

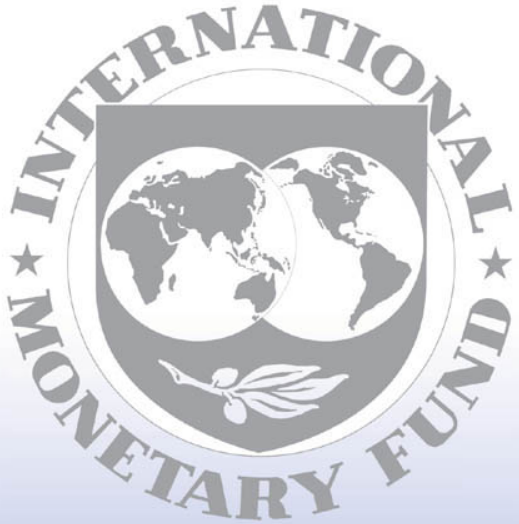

Staff

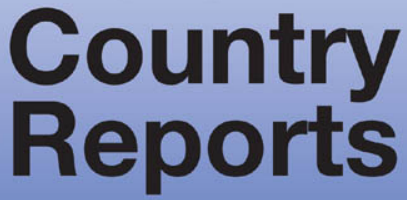




\section{Republic of Mozambique: Statistical Appendix}

This Statistical Appendix for Republic of Mozambique was prepared by a staff team of the International Monetary Fund as background documentation for the periodic consultation with the member country. It is based on the information available at the time it was completed on June 3, 2002. The views expressed in this document are those of the staff team and do not necessarily reflect the views of the government of Republic of Mozambique or the Executive Board of the IMF.

The policy of publication of staff reports and other documents by the IMF allows for the deletion of market-sensitive information.

To assist the IMF in evaluating the publication policy, reader comments are invited and may be sent by e-mail to Publicationpolicy@imf.org.

Copies of this report are available to the public from

International Monetary Fund - Publication Services

$70019^{\text {th }}$ Street, N.W. - Washington, D.C. 20431

Telephone: (202) 623-7430 - Telefax: (202) 623-7201

E-mail: publications@imf.org Internet: http://www.imf.org

Price: $\$ 15.00$ a copy

\section{International Monetary Fund \\ Washington, D.C.}




\section{INTERNATTONAL MONETARY FUND}

\section{REPUBLIC OF MOZAMBIQUE}

\section{Statistical Appendix}

Prepared by a staff team comprising David Andrews (head-AFR), Paulo Lopes (AFR), Alvaro Manoel (AFR), Wilfried Engelke (AFR), Sishir Bhattarai (AFR), Lynn Aylward (PDR), and Arnim Schwidrowski (Resident Representative)

\section{Approved by the African Department}

June 3, 2002

Contents

Page

Statistical Appendix Tables

1. Gross Domestic Product, 1996-2001 ....................................................................... 3

2. Savings and Investment, 1996-2001 ..................................................................4

3. Availability and Uses of Resources, 1996-2001,.................................................

4. Composition of Gross Domestic Product, 1996-2001 .........................................6

5. Production of Major Marketed Crops, 1996/97-2000/01 .......................................

6. Prices of Major Marketed Crops, 1996/97-2000/01 ..................................................9

7. Marketed Livestock, 1997-2001 …................................................................ 10

8. Industrial Production by Branch, 1996-2000 ….................................................. I1

9. Transport and Communications Activity, 1997-2001 .............................................. 13

10. Maputo Consumer Price Index, December 1992-April 2002 _................................... 14

11. Major Consumer Price Index (CPI) Categories, December 1997-December 2001.... 15

12. Minimum Agricultural Producer Prices, 1996/97-2000/01 ....................................... 16

13. Prices Administered by the Government, 1999-2002 ........................................... 17

14. Prices of Petroleum Products, 1998-2002 ……........................................................ 18

15. Import Prices of Oil Products, 1997-2001 ….......................................................... 19

16. Price Structure of Petroleum Products, November 2001 ............................................. 20

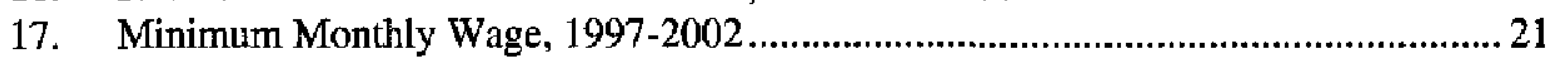

18. Expenditure on the Social Sectors as defined in the PARPA, 1999-2001 ................22

19. Number of Households Receiving Food Subsidy Assistance, March 1997.December 2001 ..................................................................24

20. Budget Subsidies to Enterprises, 1997-2001 ..................................................25

21. Government Finances (in billions of meticais) 1997-2001 ......................................26

22. Government Finances (in percent of GDP) 1997-2001 ….......................................28

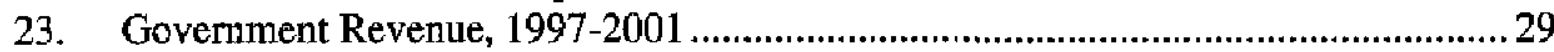


24. Locally Financed Public Investment by Sector, 1996-2000 ...............................30

25. Monetary Survey, December 1997-December 2001 ............................................31

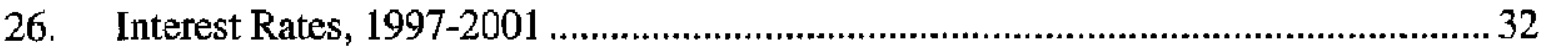

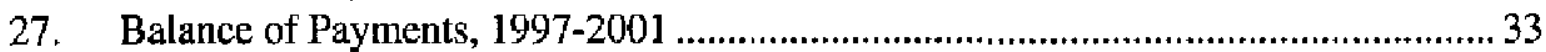

28. Foreign Trade Indicators, 1997-2001 ............................................................. 34

29. Commodity Composition of Exports, 1997-2001 .............................................. 35

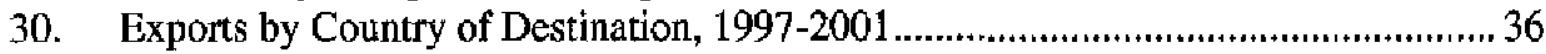

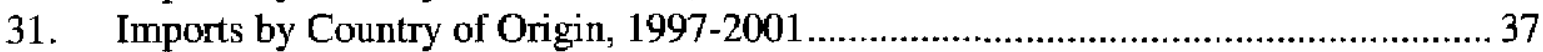

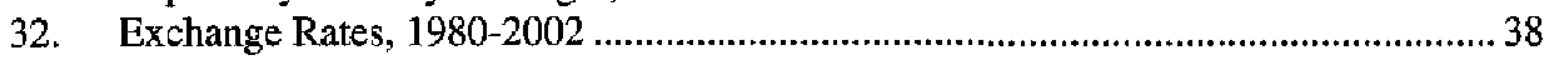


Table 1. Mozambique: Gross Domestic Product, 1996-2001

\begin{tabular}{|c|c|c|c|c|c|c|}
\hline & 1996 & 1997 & 1998 & 1999 & 2000 & 2001 \\
\hline & \multicolumn{6}{|c|}{ (In billions of meticais) } \\
\hline Total consumption & 33,317 & 39,390 & 43,714 & 47,132 & 51,916 & 55,920 \\
\hline Privale consumption & 30,692 & 35,735 & 38,816 & 40,859 & 46,540 & 51,169 \\
\hline Public consumption & 2,625 & 3,655 & 4,898 & 6,367 & 7,965 & 8,508 \\
\hline Total investrnent & 7,141 & 8,194 & 11,360 & 19,134 & 23,319 & 31,095 \\
\hline Public investment & 3,669 & 4,816 & 4,575 & 6,001 & 7,826 & 10,428 \\
\hline Private investment & 3,472 & 3,378 & 6.785 & 13,133 & 15,493 & 20,667 \\
\hline Domestic demand & 40,459 & 47,585 & 55,074 & 66,266 & 75,235 & 87,015 \\
\hline Exports of goods and nonfactor services & 3,982 & 4,510 & 4,919 & 5,256 & 7,248 & 16,380 \\
\hline Imports of goods and nonfactor services & 11,722 & 12,276 & 13,085 & 19,607 & 23,577 & 28,721 \\
\hline \multirow[t]{2}{*}{ Gross domestic praduct } & 32,719 & 39,819 & 46,908 & 51,915 & 58,905 & 74,675 \\
\hline & \multicolumn{6}{|c|}{ (In percent) } \\
\hline \multicolumn{7}{|l|}{ Real rates of change 1/ } \\
\hline Private consumption & 17.2 & 7.1 & 5.9 & 4.7 & -1.3 & -1.1 \\
\hline Public consumption & -6.3 & 21.0 & 21.0 & 9.7 & 17.4 & -4.4 \\
\hline Total investment & -8.4 & 8.4 & 32.6 & 61.4 & -9.5 & 10.3 \\
\hline Exports of goods and nonfactor services & 22.8 & 8.6 & 10.4 & -1.4 & 30.9 & 65.2 \\
\hline Imports of goods and nonfactor services & 5.0 & $1\lfloor .1$ & 8.4 & 40.4 & -3.1 & -9.7 \\
\hline Gross domestic product & 7.1 & 11.1 & 12.6 & 7.5 & 1.6 & 13.9 \\
\hline \multicolumn{7}{|l|}{ Deflators } \\
\hline Private consumptior & 42.3 & 8.8 & 2.6 & 0.6 & 15.4 & 8.2 \\
\hline Public consumption & 38.2 & 15.1 & 10.7 & 18.4 & 6.6 & 8.8 \\
\hline Investment & 23.7 & 5.8 & 4.6 & 3.8 & 20.3 & 16.4 \\
\hline Exports & 20.1 & 4.3 & $-\mathrm{L} .2$ & 8.4 & 5.3 & 33.2 \\
\hline Imports & 26.6 & 4.4 & -1.7 & 6.8 & 24.1 & 31.3 \\
\hline \multirow[t]{2}{*}{ Gross dotnestic product } & 48.2 & 9.6 & 4.6 & 2.9 & 11.7 & 11.3 \\
\hline & \multicolumn{6}{|c|}{ (In percent of GDP) } \\
\hline Total consumption & 101.8 & 98.9 & 93.2 & 90.8 & 88.1 & 74.9 \\
\hline Priwate consumption & 93.8 & 89.7 & 82.7 & 78.7 & 79.0 & 68.5 \\
\hline Public consumption & 8.0 & 9.2 & 10.4 & 12.3 & 13.5 & 11.4 \\
\hline Total investment & 21.8 & 20.6 & 24.2 & 36.9 & 39.6 & 41.6 \\
\hline Public investment & 11.2 & 12.1 & 9.8 & 11.6 & 13.3 & 14.0 \\
\hline Private investment & 10.6 & 8.5 & 14.5 & 25.3 & 26.3 & 27.7 \\
\hline Domesric demand & 123.7 & 119.5 & 117.4 & 127.6 & 127.7 & 116.5 \\
\hline Exports of goods and nonfactor services & 12.2 & 11.3 & 10.5 & 10.1 & 12.3 & 21.9 \\
\hline Imports of goods and nonfactor services & 35.8 & 30.8 & 27.9 & 37.8 & 40.0 & 38.5 \\
\hline Gross domestic product & 100.0 & 100.0 & 100.0 & 100.0 & 100.0 & 100.0 \\
\hline
\end{tabular}

Saurces: Mozambican aathorities; and staff estimates.

1) Wolume growth rates based on growth of value at previous year's prices. 
Table 2. Mozambique: Savings and investment, 1996-200:

\begin{tabular}{|c|c|c|c|c|c|c|}
\hline & 1996 & 1997 & 1998 & 1999 & 2000 & 2001 \\
\hline & \multicolumn{6}{|c|}{ (In billions of meticais) } \\
\hline Gross domestic savings (GDS) $1 /$ & -599 & 429 & 3,194 & 4,690 & 4,400 & 14,997 \\
\hline Public sector & 887 & 787 & 498 & 193 & -269 & -724 \\
\hline Private sector & $-1,486$ & -358 & 2,696 & 4,496 & 4,669 & 15,721 \\
\hline \multicolumn{7}{|l|}{ Net factor income from abroad $2 /$} \\
\hline and net unrequited transfers & 2,980 & 1,513 & 1,312 & 4,767 & 7,577 & 5,741 \\
\hline Net factor income & 442 & $-2,100$ & $-2,556$ & $-1,407$ & $-1,802$ & $-4,797$ \\
\hline Public sector & 647 & -473 & -473 & -318 & -104 & -150 \\
\hline Private sector & -171 & $-1,627$ & $-1,983$ & -873 & -669 & $-2,295$ \\
\hline Net unrequited transfers & 2,538 & 3,613 & 3,868 & 6,175 & 9,379 & 10,539 \\
\hline Public sector & 1,981 & 3,153 & 3,466 & 5,547 & 6,493 & 9,069 \\
\hline Privale sector & 556 & 460 & 402 & 628 & 2,886 & 1,470 \\
\hline Gross national savings (GNS) $3 /$ & 2,381 & 1,942 & 4,506 & 9,457 & 11,977 & 20,739 \\
\hline Public sector & .. & $\cdots$ & $\cdots$ & $\cdots$ & $\ldots$ & $\ldots$ \\
\hline Private sector & $\cdots$ & $\cdots$ & $\cdots$ & $\cdots$ & $\cdots$ & $\cdots$ \\
\hline Foreign savings $4 /$ & 4,761 & 6,252 & 6,854 & 9,677 & 11,341 & 10,356 \\
\hline Gross savings & 7,141 & 8,194 & 11,360 & 19,134 & 23,319 & 31,095 \\
\hline Gross investment & 7,141 & 8,194 & 11,360 & 19,134 & 23,319 & 31,095 \\
\hline Public sector & 3,359 & 4,263 & 4,223 & 5,475 & 7,464 & 9,859 \\
\hline \multirow[t]{2}{*}{ Private sector } & 3,782 & $3,93]$ & 7,137 & 13,659 & 15,855 & 21,236 \\
\hline & \multicolumn{6}{|c|}{ (In percent of GDP) } \\
\hline GDS $1 /$ & -1.8 & 1.1 & 6.8 & 9.0 & 7.5 & 20.1 \\
\hline Public sector & 2.7 & 2.0 & 1.1 & 0.4 & -0.5 & -1.0 \\
\hline Private sector & -4.5 & -0.9 & 5.7 & 8.7 & 7.9 & 21.1 \\
\hline \multicolumn{7}{|l|}{ Net factor income from abroad $2 t$} \\
\hline and net unnequited transferș & 9.1 & 3.8 & 2.8 & 9.2 & 12.9 & 7.7 \\
\hline Net factor income & 1.4 & -5.3 & -5.4 & -2.7 & -3.1 & -6.4 \\
\hline Net unrequited transfers & 7.8 & 9.1 & 8.2 & 11.9 & 15.9 & 14.1 \\
\hline GNS $3 \prime$ & 7.3 & 4.9 & 9.6 & 18.2 & 20.3 & 27.8 \\
\hline Public sector & $\cdots$ & $\cdots$ & $\ldots$ & $\ldots$ & $\cdots$ & $\cdots$ \\
\hline Private sector & $\cdots$ & $\cdots$ & $\cdots$ & $\cdots$ & $\cdots$ & $\cdots$ \\
\hline Forcign savings $4 /$ & 14.6 & 15.7 & 14.6 & 18.6 & 19.3 & 13.9 \\
\hline Gross savings & 21.8 & 20.6 & 24.2 & 36.9 & 39.6 & 41.6 \\
\hline Gross investment & 21.8 & 20.6 & 24.2 & 36.9 & 39.6 & 41.6 \\
\hline Public sector & 10.3 & 10.7 & 9.0 & 10.5 & 12.7 & 33.2 \\
\hline Private sector & 11.6 & 9.9 & 15.2 & 26.3 & 26.9 & 28.4 \\
\hline
\end{tabular}

Sources: Mozambican authorities; and staff estimates.

1/ GDS = GDP - total consumption = gross investment + exports of goods and nonfactor services - imports of goods and ncnfactor services.

2/ Actual external interest payments.

3) GNS = GDS + net factor income from abroad + net unrequited transfers.

4/ Exteral current account, inchuding grants (interest payments on a cash basis). 
$-5-$

Table 3. Mozambique: Availability and Uses of Resources, 1996-2001

(In percent of GDP)

\begin{tabular}{|c|c|c|c|c|c|c|}
\hline & 1996 & 1997 & 1998 & 1999 & 2000 & 2001 \\
\hline 1. GDP at market prices & 100.0 & 100.0 & 100.0 & 100.0 & 100.0 & 100.0 \\
\hline 2. Resource gap $1 /$ & 23.7 & -19.5 & -17.4 & -27.6 & -27.7 & -16.5 \\
\hline $\begin{array}{l}\text { 3. Domestic demand } \\
\text { (current sources = current uses) } 2 /\end{array}$ & 123.7 & 119.5 & 117.4 & 127.6 & 127.7 & 116.5 \\
\hline $\begin{array}{l}\text { 4. Total consumption } \\
\text { Public consumption } \\
\text { Private consumption }\end{array}$ & $\begin{array}{r}101.8 \\
8.0 \\
93.8\end{array}$ & $\begin{array}{r}98.9 \\
9.2 \\
89.7\end{array}$ & $\begin{array}{l}93.2 \\
10.4 \\
82.7\end{array}$ & $\begin{array}{l}90.8 \\
12.3 \\
78.7\end{array}$ & $\begin{array}{l}88.1 \\
13.5 \\
79.0\end{array}$ & $\begin{array}{l}74.9 \\
11.4 \\
68.5\end{array}$ \\
\hline $\begin{array}{l}\text { 5. Total savings } \\
\text { Public savings } \\
\text { Private savings }\end{array}$ & $\begin{array}{l}21.8 \\
10.3 \\
11.6\end{array}$ & $\begin{array}{r}20.6 \\
10.7 \\
9.9\end{array}$ & $\begin{array}{r}24.2 \\
9.0 \\
15.2\end{array}$ & $\begin{array}{l}36.9 \\
10.5 \\
26.3\end{array}$ & $\begin{array}{l}39.6 \\
12.7 \\
26.9\end{array}$ & $\begin{array}{l}41.6 \\
13.2 \\
28.4\end{array}$ \\
\hline 6. Foreigl savings & 14.6 & 15.7 & 14.6 & 18.6 & 19.3 & 13.9 \\
\hline $\begin{array}{l}\text { 7. Total investment } \\
\text { Public investment } \\
\text { Private investment }\end{array}$ & $\begin{array}{l}21.8 \\
10.3 \\
11.6\end{array}$ & $\begin{array}{r}20.6 \\
10.7 \\
9.9\end{array}$ & $\begin{array}{r}24.2 \\
9.0 \\
15.2\end{array}$ & $\begin{array}{l}36.9 \\
10.5 \\
26.3\end{array}$ & $\begin{array}{l}39.6 \\
12.7 \\
26.9\end{array}$ & $\begin{array}{l}41.6 \\
13.2 \\
28.4\end{array}$ \\
\hline
\end{tabular}

Sources: Tables 1 and 2.

1/ Imports of goods and nonfactor services minus exports of goods and nonfactor scrvices.

2/ $(3)=(1)+(2)=(4)+(5)$. 
Table 4. Mozambique: Composition of Gross Domestic Product, 1996-2001

\begin{tabular}{|c|c|c|c|c|c|c|}
\hline & 1996 & 1997 & 1998 & 1999 & 2000 & 2001 \\
\hline & \multicolumn{6}{|c|}{ (ln billions of meticais) } \\
\hline Agriculture & 9,973 & 12,019 & 12,758 & 13,185 & 11,914 & 14,074 \\
\hline Fishing & 1,311 & 1,566 & 1,416 & 1,305 & 1,196 & 1,436 \\
\hline Industry & 5,228 & 6,928 & 10,094 & 11,315 & $13,3,45$ & 18,539 \\
\hline Mining & 78 & 124 & 143 & 78 & 130 & 197 \\
\hline Manufacturing & 2,832 & 3,817 & 5,162 & 5,725 & 6,677 & 8,015 \\
\hline Eloctricity and water & 155 & 328 & 880 & 1,538 & 1,423 & 1,573 \\
\hline Construction & 2,163 & 2,659 & 3,908 & 3,974 & 5,114 & 8,761 \\
\hline Services & 16,207 & 19,307 & 22,640 & 26,110 & 32,450 & 40,626 \\
\hline Commerce & 7,596 & 8,974 & 10,067 & 10,942 & 11,982 & 12,852 \\
\hline Repair services & 189 & 259 & 369 & 399 & 501 & 526 \\
\hline Restaurants and hotels & 278 & 496 & 534 & 616 & 830 & 791 \\
\hline Transport and communications & 2,826 & 3,548 & 4,304 & 5,283 & 7,448 & 11,982 \\
\hline Financial services & 1,221 & 1,284 & 1,229 & 1,098 & 2,327 & 3,206 \\
\hline Rcal estate rentals & 879 & 1,035 & 1,120 & 1,145 & 1,143 & 1,231 \\
\hline Corporate services & 414 & 458 & 601 & 435 & 474 & 521 \\
\hline Government services & 1,452 & 1,793 & 2,385 & 3,554 & 4,211 & 5,320 \\
\hline Other services & 1,353 & 1,461 & 2,030 & 2,637 & 3,534 & 4,198 \\
\hline \multirow[t]{2}{*}{ Gross domestic product } & 32,719 & 39,819 & 46,908 & 51,915 & 58,905 & 74,675 \\
\hline & \multicolumn{6}{|c|}{ (In percent of gross domestic product) } \\
\hline Agriculture & 30.5 & 30.2 & 27.2 & 25.4 & 20.2 & 18.8 \\
\hline Fishing & 4.0 & 3.9 & 3.0 & 2.5 & 2.0 & 1.9 \\
\hline Itdustry & 16.0 & 17.4 & 21.5 & 21.8 & 22.7 & 24.8 \\
\hline Mining & 0.2 & 0.3 & 0.3 & 0.1 & 0.2 & 0.3 \\
\hline Manufacturing & 8.7 & 9.6 & 11.0 & 11.0 & 11.3 & 10.7 \\
\hline Electricity and water & 0.5 & 0.8 & 19 & 3.0 & 2.4 & 2.1 \\
\hline Construction & 6.6 & 6.7 & 8.3 & 7.7 & 8.7 & 11.7 \\
\hline Services & 49.5 & 48.5 & 48.3 & 50.3 & 55.1 & 54.4 \\
\hline Commerce & 23.2 & 22.5 & 21.5 & 21.1 & 20.3 & 17.2 \\
\hline Repair services & 0.6 & 0.6 & 0.8 & 0.8 & 0.9 & 0.7 \\
\hline Restauratits and hotels & 0.8 & 1.2 & 1.1 & 1.2 & 1.4 & 1.1 \\
\hline Transport and communications & 8.6 & 8.9 & 9.2 & 10.2 & 12.6 & 16,0 \\
\hline Financial services & 3.7 & 3.2 & 2.6 & 2.1 & 4.0 & 4,3 \\
\hline Real estate rentals & 2.7 & 2.6 & 2.4 & 2.2 & $\llbracket .9$ & 1.6 \\
\hline Corporate services & 1.3 & 1.1 & 1.3 & 0.8 & 0.8 & 0.7 \\
\hline Governmeni services & 4.4 & 4.5 & 5.1 & 6.8 & 7.1 & 7.1 \\
\hline Other services & 4.1 & 3.7 & 4,3 & 5.1 & 6,0 & 5.6 \\
\hline Gross domestic product & 100.0 & 100.0 & 100.0 & 100.0 & 100.0 & 100.0 \\
\hline
\end{tabular}


Table 4, Mozambique: Composition of Gross Domestic Product, 1996-2001 (concluded)

\begin{tabular}{|c|c|c|c|c|c|c|}
\hline & 1996 & 1997 & 1998 & $\$ 999$ & 2000 & 2001 \\
\hline & \multicolumn{6}{|c|}{ (Annual percentage change) } \\
\hline \multicolumn{7}{|l|}{ Real rates of change $1 /$} \\
\hline Agriculture & $\cdots$ & 9.4 & 9.6 & 6.5 & -10.3 & 10.0 \\
\hline Fishing & $\ldots$ & 2.1 & -11.3 & -1.9 & -10.3 & 10.6 \\
\hline Mining & $\cdots$ & 21.1 & 20.2 & -6.3 & -29.4 & 11.8 \\
\hline Manufacturing & $\ldots$ & 31.8 & 13.6 & 15.0 & 11.0 & 30.3 \\
\hline Electricity and water & $\cdots$ & 37.9 & 252.4 & 92.4 & .7 .7 & 8.0 \\
\hline Construction & $\ldots$ & 18.1 & 26.1 & 3.5 & 1.5 & 53.6 \\
\hline Commerce & $\cdots$ & 8.0 & 11.4 & 2.8 & 4.4 & 7.4 \\
\hline Repair services & $\cdots$ & 23.5 & 16.6 & 3.3 & 2.8 & 1.2 \\
\hline Restaurants and hotels & $\ldots$ & 35.5 & .2 .4 & 5.5 & 5.3 & -10.1 \\
\hline Transport and communications & $\ldots$ & 17.3 & 4.5 & 9.2 & 0.1 & 35.5 \\
\hline Financial services & $\ldots$ & 29.9 & -17.4 & -23.3 & 84.3 & 2.1 \\
\hline Real estate rentals & $\ldots$ & 3.3 & 3.8 & 2.2 & 3.7 & 2.4 \\
\hline Corporate services & $\ldots$ & 0.1 & 20.4 & 4.6 & -1.7 & 7.3 \\
\hline Government services & $\ldots$ & 2.2 & 14.7 & 4.5 & 5.9 & 11.4 \\
\hline OAher services & $\cdots$ & 13.1 & 22.5 & 27.3 & 19.4 & 8.4 \\
\hline Gross domestic product & $+\cdot \cdot$ & 11.1 & 12.6 & 7.5 & 1.6 & 13.9 \\
\hline \multicolumn{7}{|l|}{ Deflators } \\
\hline Agriculture & $\ldots$ & 9.6 & 4.6 & 0.0 & 11.7 & 11.3 \\
\hline Fishing & $\cdots$ & 17.1 & 2.0 & -6.1 & 2.2 & 3.7 \\
\hline Mining & $\cdots$ & 32.4 & -4.1 & -42.2 & 138.1 & 3.6 \\
\hline Manufacturing & $\cdots$ & 2.3 & 19.0 & -3.6 & 5.1 & 5.7 \\
\hline Electricity and water & $\ldots$ & 53.2 & -23.8 & -9.2 & 0.2 & 5.3 \\
\hline Construction & $\ldots$ & 4.0 & 16.6 & -1.7 & 26.7 & 22.9 \\
\hline Commerce & $\cdots$ & 9.4 & 0.7 & 5.7 & 4.9 & -0.3 \\
\hline Repair services & $\ldots$ & 10.6 & 22.4 & 4.7 & 22.3 & 1.7 \\
\hline Restaurants and hotels & $\ldots$ & 31.7 & 10.4 & 9.3 & 27.9 & 6.0 \\
\hline Transport and communications & $\ldots$ & 7.0 & 16.1 & 12.4 & 40.9 & 28.0 \\
\hline Financial services & $\cdots$ & -19.0 & 16.0 & 16.5 & 14.9 & 7.3 \\
\hline Real estate rentals & $\ldots$ & 14.0 & 4.3 & 0.1 & -3.8 & 2.4 \\
\hline Corporate services & $\ldots$ & 10.6 & 9.1 & -30.8 & 10.8 & 0.4 \\
\hline Government services & $\ldots$ & 20.9 & 15.9 & 42.6 & 11.9 & 13.5 \\
\hline Other services & $\ldots$ & 0.1 & 14.2 & 2.3 & 21.7 & 52.7 \\
\hline Gross domestic product & $\ldots$ & 9.6 & 4.6 & 2.9 & 11.7 & 11.3 \\
\hline
\end{tabular}

Source: Mozambican guthorities.

U Volume growth rates based on growth of vahue at previous year's prices. 
Table 5. Mozambique: Production of Major Marketed Crops, 1996/97-2000/01

\begin{tabular}{|c|c|c|c|c|c|}
\hline & $1996 / 97$ & $1997 / 98$ & $1998 / 99$ & $1999 / 00$ & $2000 / 01$ \\
\hline & \multicolumn{5}{|c|}{ (In thousends of toms) } \\
\hline Export crops & 443.5 & 559.1 & 694.9 & 549.2 & $1,207.6$ \\
\hline Cotton & 74.0 & 91.0 & 106.7 & 32.3 & 77.8 \\
\hline Cogra & 35.6 & 36,0 & 44.4 & 44.0 & 64.0 \\
\hline Tea (leaf) & 1.5 & 1.5 & 5.4 & 10.5 & 11.1 \\
\hline Sugarcane & 278.9 & 368.7 & 469.5 & 3972 & 983.3 \\
\hline Cashew nuts & 43.3 & 51.7 & 58.7 & 51.9 & 58.1 \\
\hline Citrus & 10.2 & 102 & 10.2 & 13.3 & 13.3 \\
\hline Basic food crops & 488.7 & 504.4 & 576.5 & 494.5 & 564.6 \\
\hline Maize & 256.3 & 270.2 & 304.1 & 248.8 & 287.6 \\
\hline Rice & 24.9 & 26.7 & 28.9 & 23.5 & 27.7 \\
\hline Sorghum & 4.4 & 4.1 & 5.7 & 4.4 & 5.5 \\
\hline Cassava & 76.1 & 74.8 & 83.8 & 81.0 & 95.3 \\
\hline Peanuts & 23.4 & 23.8 & 26,4 & 20.6 & 19.8 \\
\hline Beans & 45.0 & 45.7 & 68.1 & 52.8 & 59.8 \\
\hline Vegetables & 50.0 & 50.5 & 51.0 & 54.5 & 66.2 \\
\hline Onions & 8.5 & 8.5 & 8.5 & 9.0 & 2.7 \\
\hline Industria! inputs & 382 & 38.9 & 38.1 & 17.3 & 23.4 \\
\hline Sisa! & 24.0 & 24.0 & 24.0 & & $\ldots$ \\
\hline Tobacco & 0.7 & 0.7 & 0.7 & 4.2 & 8.5 \\
\hline Mafurra & 0.7 & 1.5 & 0.7 & 0.3 & 0.5 \\
\hline Tomatoes & 12.2 & 12.2 & 12.2 & 12.4 & 129 \\
\hline \multirow[t]{2}{*}{ Sunflowers } & 0.5 & 0.5 & 0.6 & 0.4 & 6.4 \\
\hline & \multicolumn{5}{|c|}{ (In billions of meticais, unless otherwise indicated) } \\
\hline Export crops & 552.7 & 690.2 & 773.5 & 529.1 & 964.3 \\
\hline Cotton & 241.2 & 308.5 & 341.9 & 118.3 & 336.3 \\
\hline Copra & 68.6 & 69.5 & 75.6 & 85.6 & 147.0 \\
\hline Tea (leaf) & 1.5 & $\mathrm{t} .5$ & 0.5 & 1.1 & 1.4 \\
\hline Sugarcane & 41.8 & 55.3 & 69.1 & 61.2 & 179.0 \\
\hline Cashew nuts & 173.3 & 227.6 & 258.6 & 224.0 & 260.2 \\
\hline Citrus & 26.2 & 27.8 & 27.8 & 38.9 & 40.4 \\
\hline Basic food crops & 859.6 & 931.1 & $1,143.9$ & $1,037.9$ & $1,188.8$ \\
\hline Maize & 307.6 & 354.1 & 456.6 & 356.3 & 427.4 \\
\hline Rice & 97.7 & 105.2 & 72.3 & 61.6 & 75.4 \\
\hline Sorghum & 9.4 & 8.8 & 12.3 & 10.0 & 12.9 \\
\hline Cassava & 60.2 & 67.3 & 121.3 & 122.7 & 149.9 \\
\hline Peanuts & 94.1 & 95.9 & 119.1 & 1003 & 100.0 \\
\hline Beants & 148.6 & 150.9 & 224.8 & 192.9 & 226.6 \\
\hline Vegetables & 104.6 & 117.0 & 106.9 & 143.5 & 180.8 \\
\hline Onions & 37.4 & 31.9 & 30.6 & 50.6 & 15.9 \\
\hline Industrial inputs & 56.9 & 49.8 & 57.9 & 118.7 & 228.8 \\
\hline Sisal & 1.1 & 1.1 & 1.1 & $\ldots$ & $\ldots$ \\
\hline Tobacco & 9.2 & 9.6 & 9.2 & 63.3 & 150.8 \\
\hline Mafurra & 0.4 & 0.7 & 0.3 & 0.1 & 0.2 \\
\hline Totnatoes & 44.9 & 36.9 & 45.8 & 54.2 & 58.7 \\
\hline Sunflowers & 1.4 & 1.5 & 1.5 & 1.1 & 19.0 \\
\hline Total production & $1,469.2$ & $1,671.0$ & $1,975.3$ & $1,685.8$ & $2,381.9$ \\
\hline \multicolumn{6}{|l|}{ Memorandum item: } \\
\hline $\begin{array}{l}\text { Total production (in } \\
\text { U.S. doilars) } /\end{array}$ & 127.3 & 141.0 & 155.7 & 186.8 & 264.0 \\
\hline
\end{tabular}

Source: Ministry of Planning and Finance.

1) Market exchange rates uscd for all crop years. 
Table 6. Mozambique: Prices of Major Marketed Crops,

1996/97-2000/01

\begin{tabular}{|c|c|c|c|c|c|}
\hline & $1996: 97$ & $1997 / 98$ & $1998 / 99$ & $1999 / 00$ & 200001 \\
\hline & \multicolumn{5}{|c|}{ (In thousands of meticais per ton) } \\
\hline \multicolumn{6}{|l|}{ Export erops } \\
\hline Colton & 3,260 & 3,390 & 3,203 & 3,659 & 4,323 \\
\hline Copra & 1,930 & 1,930 & 1,702 & 1,945 & 2,297 \\
\hline Tea (leaf) & 100 & 100 & 92 & 106 & 125 \\
\hline Sugar & 150 & 150 & 147 & 154 & 182 \\
\hline Cashew nuts & 4,000 & 4,400 & 4,404 & 4,317 & 4,479 \\
\hline \multicolumn{6}{|l|}{ Bassic food crops } \\
\hline Maize & 1,200 & 1,310 & 1,502 & 1,432 & 1,486 \\
\hline Rice & 3,920 & 3,936 & 2,503 & 2,619 & 2,718 \\
\hline Sorghum & 2,140 & 2,140 & 2,142 & 2,259 & 2,344 \\
\hline Cassava & 790 & 901 & 1,447 & 1.516 & 1,572 \\
\hline Peanuts & 4.030 & 4,030 & 4,505 & 4,877 & 5,060 \\
\hline Citrus & 2,570 & 2,732 & 2735 & 2,927 & 3,037 \\
\hline Beans & 3,300 & 3,300 & 3,303 & 3,654 & 3,791 \\
\hline Vegetables & 2,093 & 2,315 & 2,095 & 2,633 & 2,731 \\
\hline Onions & 4,400 & 3,749 & 3.603 & 5.627 & 5,838 \\
\hline \multicolumn{6}{|l|}{ Other crops } \\
\hline Sisal & 46 & 46 & 46 & $\ldots$ & $\ldots$ \\
\hline Tobacco & $13, \mathrm{j} 67$ & 13,693 & 13,180 & 15,009 & 17,822 \\
\hline Mafura & 475 & 475 & 484 & 449 & 466 \\
\hline Tomatoes & 3,678 & 3,027 & 3,752 & 4,374 & 4,538 \\
\hline \multirow[t]{2}{*}{ Sunflowers } & 2,600 & 2,704 & 2,603 & 2,855 & 2,962 \\
\hline & \multicolumn{5}{|c|}{ (Annual percontage changes. unless ptherwise indicated) } \\
\hline \multicolumn{6}{|l|}{ Export crops } \\
\hline Cotton & -15.3 & 4.0 & $-5,5$ & 14.2 & 18.1 \\
\hline Copra & 10.3 & 0.0 & $-1 \perp .8$ & 14.2 & 18.1 \\
\hline Tea (leaf) & 17.6 & 0.0 & -8.0 & 14.9 & 18.1 \\
\hline Sugat & 7.1 & 0.0 & -2.0 & 4.8 & 18.1 \\
\hline Cashew nuts & 14.3 & 10.0 & 0.1 & -2.0 & 3.8 \\
\hline \multicolumn{6}{|l|}{ Basic foud crops } \\
\hline Maize & -17.8 & 9.2 & 14.7 & -4.6 & 3.7 \\
\hline Rice & 100.0 & 0.4 & -36.4 & 4.6 & 3.8 \\
\hline Sorghum & 10.3 & 0.0 & 0.1 & 5.5 & 3.8 \\
\hline Cassava & 17.9 & 14.1 & 60.6 & 4.7 & 3.8 \\
\hline Peanuts & 8.9 & 0.0 & 11.8 & 8.3 & 3.8 \\
\hline Citrus & 6.6 & 6.3 & 0.1 & 7.0 & 3.8 \\
\hline Beans & -19.1 & 0.0 & 0.1 & 10.6 & 3.8 \\
\hline Vegetables & 0.0 & 10.6 & -9.5 & 25.7 & 3.8 \\
\hline Onions & 7.1 & -14.8 & -3.9 & 56.2 & 3.8 \\
\hline \multicolumn{6}{|l|}{ Other crops } \\
\hline Sisal & 0.0 & 0.0 & 0.0 & $\ldots$ & $\ldots$ \\
\hline Tobacco & 8.6 & 4.0 & -3.7 & 13.9 & 18.7 \\
\hline Mafura & 6.0 & 0.0 & 1.9 & -7.3 & 3.8 \\
\hline Tomatoes & 6.0 & -17.7 & 24.0 & 56.6 & 3.8 \\
\hline Sunfowers & 0.0 & 4.0 & -3.7 & 9.7 & 3.8 \\
\hline \multicolumn{6}{|l|}{ Memvrandum iterrs: } \\
\hline Private consumption prices & 11.2 & 0.2 & 1.0 & $\ldots$ & $\ldots$ \\
\hline \multicolumn{6}{|l|}{ Official exchange rate } \\
\hline (meticais per U.S. dollar, period average) & 2.3 & 4.0 & 6.9 & 19.5 & 36.8 \\
\hline
\end{tabular}

Sources: Ministry of Agriculture; and Ministry of Planning and Finance. 
Table 7. Mozambique; Marketed Livestock, 1997-2001

\begin{tabular}{|c|c|c|c|c|c|}
\hline & 1997 & 1998 & 1999 & 2000 & 2001 \\
\hline Production volume & \multicolumn{5}{|c|}{ (In units stated) } \\
\hline Beef (in tons) & 882 & 1,140 & 1,050 & 1,399 & 1,093 \\
\hline Pork (in tons) & 281 & 296 & 385 & 226 & 349 \\
\hline Chicken (in tons) & 5,109 & 4,579 & 5,417 & 4,784 & 5,193 \\
\hline Eggs (in thousands) & 6,982 & 4,945 & 5,440 & 5,440 & 12,824 \\
\hline Milk (in thousands of liters) & 821 & 742 & 915 & 915 & 781 \\
\hline Production value & \multicolumn{5}{|c|}{ (In millions of meticais) } \\
\hline Beef & 12,608 & 17,927 & 17,501 & 23,911 & 19,494 \\
\hline Pork & 4,017 & 4,655 & 6,414 & 3,919 & 6,287 \\
\hline Chicken & 66,080 & 65,190 & 81,697 & 73,158 & 82,391 \\
\hline Eggs & 4,515 & 3,516 & 4,100 & 4,364 & 10,673 \\
\hline Milk & 2,515 & 2,501 & 3.268 & 3,726 & 3,298 \\
\hline Total & 89,735 & 93,789 & 112,980 & 109,077 & 122,143 \\
\hline Average prices & \multicolumn{5}{|c|}{ (In units stated) } \\
\hline Beef $1 /$ & 14,295 & 15,725 & 16,668 & 17,194 & 17,839 \\
\hline Pork $\mathrm{l} /$ & 14,295 & 15,726 & 16,660 & 17,366 & 18,017 \\
\hline Chicken $1 /$ & 12,934 & 14,237 & 15,082 & 15,291 & 15,865 \\
\hline Eggs $2 /$ & 647 & 711 & 754 & 802 & 832 \\
\hline Milk $3 /$ & 3,063 & 3,371 & 3,572 & 4,072 & 4,225 \\
\hline
\end{tabular}

Sources: Ministry of Agriculture; and Ministry of Planning and Finance.

1/ Meticais per kilogram.

2/ Meticais per unit.

$3 i$ Meticais per liter. 
Table 8. Mozambique: Industrial Production by Branch, 1996-2000

\begin{tabular}{|c|c|c|c|c|c|}
\hline & 1996 & 1997 & 1998 & 1999 & $200 \circ$ \\
\hline & \multicolumn{5}{|c|}{ (Current prices; in millions of meticais) } \\
\hline Industry and fisheries & $5,974,994$ & $7,815,079$ & $8,114,110$ & $9,478,376$ & $11,152,741$ \\
\hline Fisheries & $1,783,574$ & $2,049,304$ & $1,803,387$ & $1,691,552$ & $1,631,167$ \\
\hline Mining & 182,268 & 247,100 & 275,850 & 205,311 & 228,339 \\
\hline Manufacturing & $3,464,561$ & $4,765,220$ & $4,749,215$ & $5,717,538$ & $7,575,713$ \\
\hline Food processing & 858,178 & $1,100,000$ & $1,029,377$ & $1,189,610$ & $1,187,585$ \\
\hline \multicolumn{6}{|l|}{ Processed animal feed, } \\
\hline Beverages & 641,870 & 857,000 & $1,133,889$ & $1,604,142$ & $1,699,912$ \\
\hline Tobacco & 90,828 & 118,000 & 124,978 & 134,269 & 142,285 \\
\hline Textiles & 342,904 & 589,000 & 151,299 & 403,876 & 330,036 \\
\hline Clothing & 92,678 & 200,000 & 219,910 & 236,873 & 193,566 \\
\hline Footwear & 3,370 & 1,000 & 4,330 & 5,834 & $\ldots$ \\
\hline Wood and cork & 222,316 & 231,000 & 258,000 & 260,586 & 344,332 \\
\hline Furniture & 6,372 & 7,000 & 4,692 & 6,785 & 7,894 \\
\hline Paper & 32,562 & 317,000 & 379,860 & 402,239 & 448,162 \\
\hline Chemical products & 41,661 & 44,000 & 46,633 & 35,303 & 38,837 \\
\hline Other chemicals & 161,296 & 197,000 & 210,847 & 224,230 & 246,678 \\
\hline Oil refmeries & 20,133 & 27,000 & 29,000 & 31,320 & 31,626 \\
\hline Rubber & 150,243 & 168,000 & 143,035 & 72,425 & 79,676 \\
\hline Plastics & 34,543 & 55,000 & 69,093 & 124,450 & 136,909 \\
\hline Glass & 2,824 & 2,000 & 24,125 & 24,193 & 19,402 \\
\hline Other nonmetallic mineral products & 364,469 & 438,220 & 568,898 & 834,960 & 800,833 \\
\hline Metalworking (iron and steel) & 37,231 & 16,000 & 31,715 & 7,578 & 8,169 \\
\hline Metalworking (noniron) & 6,929 & 6,000 & 3,168 & 4,734 & 3,816 \\
\hline Metallurgy (except machinery) & 16,619 & 163,000 & 85,161 & 66,343 & $1,807,207$ \\
\hline of which : aluminum & $\ldots$ & $\ldots$ & $\ldots$ & $\ldots$ & $1,721,515$ \\
\hline Nonelectrical machinery & 2,654 & 7,000 & 7,095 & 5,117 & 5,183 \\
\hline Appliances and electrical machinery & 27,779 & 23,000 & 31,022 & 31,073 & 31,473 \\
\hline Transpon machinery & 86,115 & 29,000 & 58,211 & 7,911 & 8,013 \\
\hline Other manufacturing & 5,741 & 6,000 & 4,877 & 3,687 & 4,118 \\
\hline Electricity & 544,591 & 753,455 & $1,285,658$ & $1,863,975$ & $1,717,521$ \\
\hline
\end{tabular}


Table 8. Mozambique: Industrial Production by Branch, 1996-2000 (concluded)

\begin{tabular}{|c|c|c|c|c|c|}
\hline & 1996 & 1997 & 1998 & 1999 & 2000 \\
\hline & \multicolumn{5}{|c|}{ (In percent of GDP) } \\
\hline Industry and fisheries & 18.3 & 19.6 & 17.3 & 18.3 & 18.9 \\
\hline Fisheries & 5.5 & 5.1 & 3.8 & 3.3 & 2.8 \\
\hline Mining & 0.6 & 0.6 & 0.6 & 0.4 & 0.4 \\
\hline Manufacturing & 10.6 & 12.0 & 10.1 & 11.0 & 12.9 \\
\hline Food processing & 2.6 & 2.8 & 2.2 & 2.3 & 2.0 \\
\hline $\begin{array}{l}\text { Processed animal feed, } \\
\text { tea, and cashew nuts }\end{array}$ & \multicolumn{4}{|c|}{ Processed animal feed, } & $\ldots$ \\
\hline Beverages & 2.0 & 2.2 & 2.4 & 3.1 & 2.9 \\
\hline Tobacco & 0.3 & 0,3 & 0.3 & 0.3 & 0.2 \\
\hline Textiles & 1.0 & 1.5 & 0.3 & 0.8 & 0.6 \\
\hline Clothing & 0.3 & 0.5 & 0.5 & 0.5 & 0.3 \\
\hline Footwear & 0.0 & 0.0 & 0.0 & 0.0 & 0.0 \\
\hline Wood and cork & 0.7 & 0.6 & 0.6 & 0.5 & 0.6 \\
\hline Furniture & 0.0 & 0.0 & 0.0 & 0.0 & 0.0 \\
\hline Paper & 0.1 & 0.8 & 0.8 & 0.8 & 0.8 \\
\hline Chemical products & 0.1 & 0.1 & 0.1 & 0.1 & 0.1 \\
\hline Other chemicals & 0.5 & 0.5 & 0.4 & 0.4 & 0.4 \\
\hline Oil refineries & 0.1 & 0.1 & 0.1 & 0.1 & 0.1 \\
\hline Rubber & 0.5 & 0.4 & 0.3 & 0.1 & 0.1 \\
\hline Plastics & 0.1 & 0.1 & 0.1 & 0.2 & 0.2 \\
\hline Glass & 0.0 & 0.0 & 0.1 & 0.0 & 0.0 \\
\hline Other nonmetallic minerak products & 1.1 & 1.1 & 1.2 & 1.6 & 1.4 \\
\hline Metalworking (iron and steel) & 0.1 & 0.0 & 0.1 & 0.0 & 0.0 \\
\hline Metalworking (noniton) & 0.0 & 0.0 & 0.0 & 0.0 & 0.0 \\
\hline Metallurgy (except machinery) & 0.1 & 0.4 & 0.2 & 0.1 & 3.1 \\
\hline Of which : aluminum & $\ldots$ & $\ldots$ & $\ldots$ & $\ldots$ & 2.9 \\
\hline Nonelectrical machinery & 0.0 & 0.0 & 0.0 & 0.0 & 0.0 \\
\hline Appliances and electrical machinery & 0.1 & 0.1 & 0.1 & 0.1 & 0.1 \\
\hline Transport machinery & 0.3 & 0.1 & 0.1 & 0.0 & 0.0 \\
\hline Other manufacturing & 0.0 & 0.0 & 0.0 & 0.0 & 0.0 \\
\hline Electricity & 1.7 & 1.9 & 2.7 & 3.6 & 2.9 \\
\hline
\end{tabular}

Source: Mozambican authorities; and staff estimates. 
Table 9. Mozambjquc: Transport and Communications Activity, 1997-200]

(In units indicated)

\begin{tabular}{|c|c|c|c|c|c|}
\hline & 1997 & 1998 & 1999 & 2000 & 2001 \\
\hline \multicolumn{6}{|l|}{ Freight transport } \\
\hline \multicolumn{6}{|l|}{ Rail } \\
\hline In millions of ron-kiloneters & 899.2 & 860.9 & 721.2 & 604.8 & 774.5 \\
\hline In billions of meticais & 630.1 & 584.2 & 532.8 & 462.2 & 591.9 \\
\hline Unit tariff $/ /$ & 700.7 & 678.6 & 738.8 & 764.2 & 764.2 \\
\hline \multicolumn{6}{|l|}{ Road } \\
\hline In millions of ton-kilometers & 360.9 & 175.1 & 233.5 & 22.4 .2 & 245.3 \\
\hline In billions of meticais & 124.6 & 140.1 & 233.5 & 207.8 & 227.4 \\
\hline Unit tariff $1 /$ & 774.4 & 800.1 & $1,000.0$ & 926.9 & 926.9 \\
\hline \multicolumn{6}{|l|}{ Maritime } \\
\hline In millions of ton-kilometers & 118.4 & 113.1 & 201.0 & 202.8 & 247.3 \\
\hline In billions of meticais & 37.6 & 37.3 & 69.6 & 97.3 & 118.7 \\
\hline Unit tariff $1 /$ & 317.6 & 329.8 & 346.3 & 480.0 & 480.0 \\
\hline \multicolumn{6}{|l|}{ Air } \\
\hline In miltions of ton-kilometers & 5.5 & 5.9 & 6.1 & 7.2 & 6.7 \\
\hline In billions of meticais & 22.8 & 24.8 & 26.9 & 31.8 & 29.5 \\
\hline Unit tariff $1 /$ & $4,145.5$ & $4,203.4$ & $4,409.8$ & $4,410.0$ & $4,410.0$ \\
\hline \multicolumn{6}{|l|}{ Port throughput } \\
\hline In thousands of shipping tons & $8,959,9$ & $7,606.0$ & $6,117.6$ & $6,096.7$ & $7,311.8$ \\
\hline In billions of meticais & 573.5 & $\$ 32.4$ & 449.6 & 595.6 & 714.4 \\
\hline Unit tariff $2 /$ & 64.0 & 70.0 & 73.5 & 97.7 & 97.7 \\
\hline \multicolumn{6}{|l|}{ Oil pipeline throughput } \\
\hline In millions of ton-kilometers & 329.7 & 355.9 & 324.0 & 232.7 & 234.4 \\
\hline In billions of meticais & 222.4 & 244.5 & 226.8 & 208.3 & 345.6 \\
\hline Unit tariff $1 /$ & 674.6 & 687.2 & 700.0 & 895.2 & $1,474.2$ \\
\hline Total freight receipts $3 /$ & $1,611.0$ & 1563.3 & $1,539.2$ & $1,503.1$ & $2,027.4$ \\
\hline \multicolumn{6}{|l|}{ Passenger transport } \\
\hline \multicolumn{6}{|l|}{ Rail } \\
\hline In miltions of passenger-kilometers & 403.1 & 155.0 & 144.7 & 129.8 & 142.0 \\
\hline In billions of meticais & 39.8 & 20.0 & 18.4 & 16.9 & 18.5 \\
\hline Unit tariff 4 & 98.7 & 129.0 & 127.2 & 130.0 & 130.0 \\
\hline \multicolumn{6}{|l|}{ Road } \\
\hline In millions of passenger-kilometers & $20,773.2$ & $25,625.5$ & $29,023.8$ & $27,029.0$ & $36,680.5$ \\
\hline In billions of meticais & $1,641.2$ & $1,993.0$ & $2,292.6$ & $2,679.2$ & $5,202.5$ \\
\hline Lnit tariff 4 & 79.0 & 77.8 & 79.0 & 99.1 & 141.8 \\
\hline \multicolumn{6}{|l|}{ Air } \\
\hline In mijlions of passenger-kiloneters & 291.7 & 311.7 & 329.8 & 377.6 & 272.4 \\
\hline In billions of meticais & 297.3 & 333.5 & 352.9 & 444.4 & 320.6 \\
\hline Unit tariff 4 & $1,019.2$ & $1,069.9$ & $\$, 070.0$ & $I, 177,0$ & $1,177.0$ \\
\hline \multicolumn{6}{|l|}{ Maritine } \\
\hline In millions of passenger-kilometers & 6.6 & 6.0 & 2.1 & 2.2 & 2.3 \\
\hline In tillions of meticais & 4.5 & 4.2 & 1.5 & 3.3 & 3.9 \\
\hline Unit tariff 4 & 681.8 & 7000 & 714.3 & $1,514.6$ & $1,697.1$ \\
\hline Total passenger receipts $3 /$ & $1,982.8$ & $2,350.7$ & $2,665,4$ & $3,143.9$ & $5,545.5$ \\
\hline Cornmunications receipts $3 /$ & $857 . i$ & 925.3 & 111.4 & $1,748.1$ & $2,489.0$ \\
\hline $\begin{array}{l}\text { Total transport and communications } \\
\text { receipts } 3 \text { / }\end{array}$ & $4,450.9$ & $4,839.3$ & $4,316.0$ & $6,495.1$ & $10,061.9$ \\
\hline
\end{tabular}

Source: Mozambican authonities.

1/ Meticais per ton-kilometer.

2/ In millions of meticais per ton.

$3 /$ In billions of meticais.

4/ Meticais per passenger-kilometer. 
Table 10. Mozambique: Maputo Consumer Price Index, December 1992-April 2002

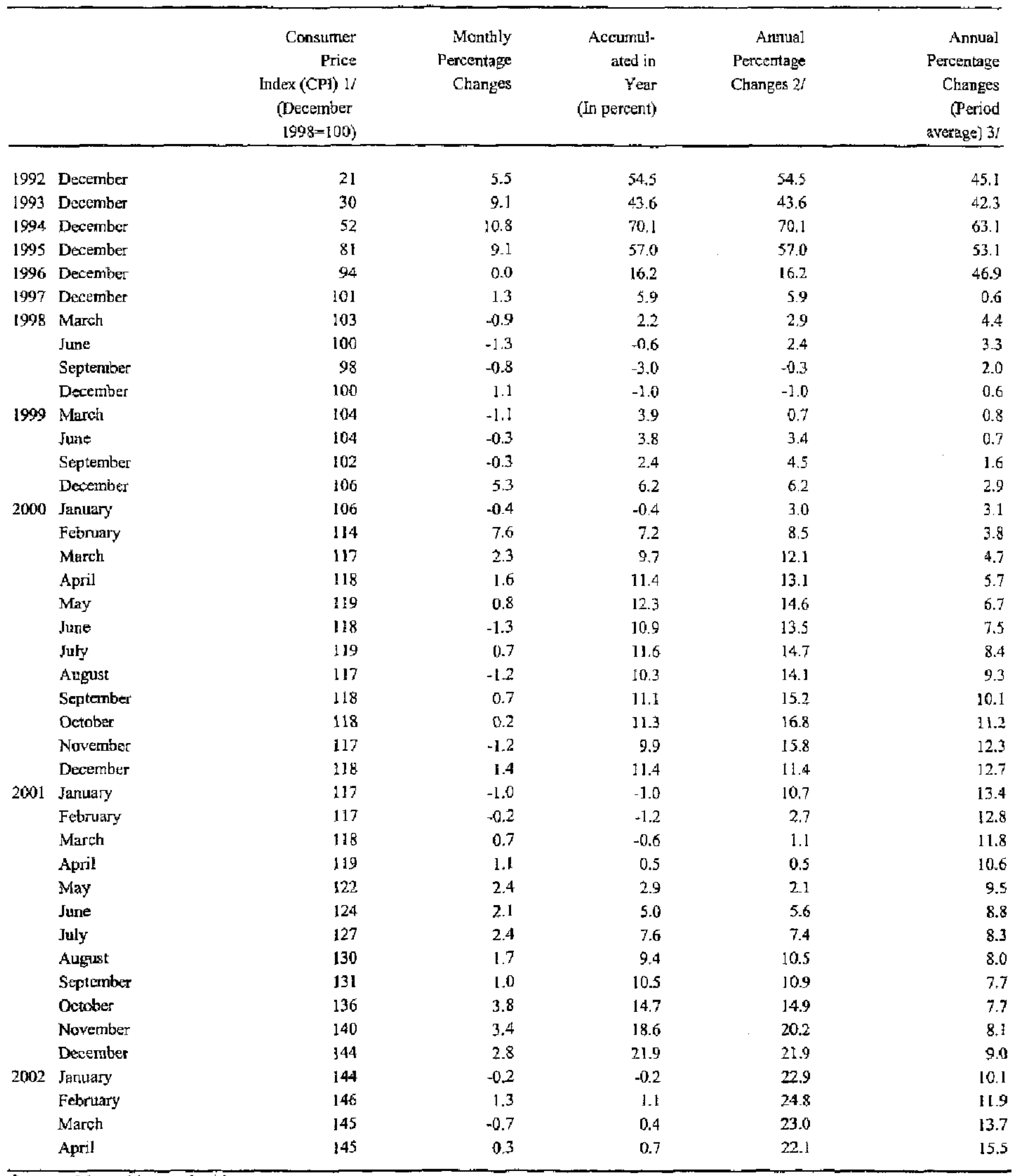

Source: Mozambican authorities.

1/ In February 2000, the National Statistics Institute (INE) began compiling a new CPI seties with December 1998 as base period. The consumer price index (CPI) was rebased on weights stemming from the 1998 census. The new index was extended backward using the growth rates of the previous CPI.

2/ Compared with same month one year earlier.

3/ Monthy average of the previous 12 months. 
Table 11. Mozambique: Major Consumer Price Index (CPI) Categories,

December 1997-Deccmber 2001

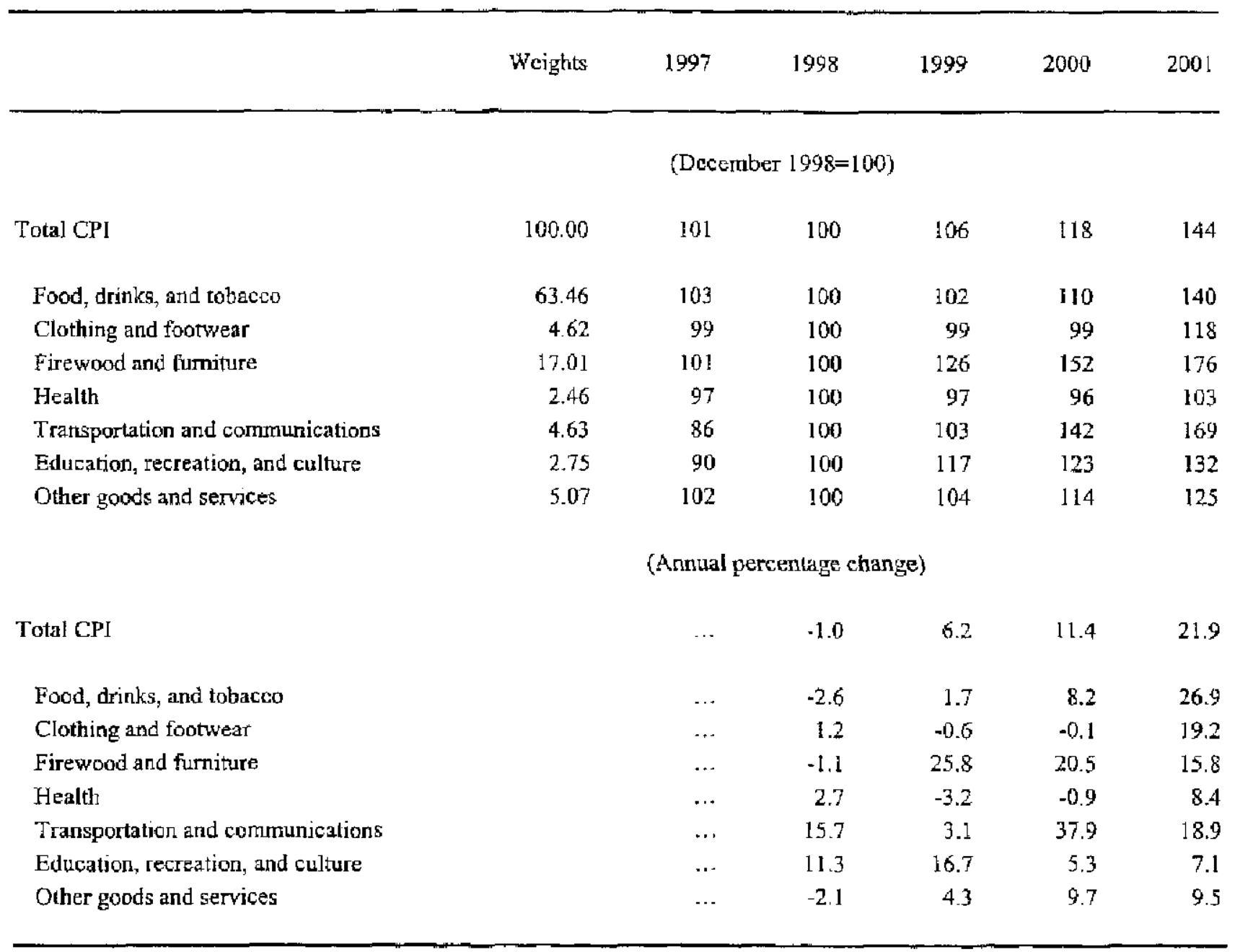

Source: Mozambican authorities; and staff estimates. 
Table 12. Mozambique: Minimum Agricultural Producer Prices 1996/97-2000/01 1/2/

\begin{tabular}{|c|c|c|c|c|c|}
\hline & $1996: 97$ & $1997 / 98$ & $1998 / 99$ & $1999 / 00$ & $2000 / 01$ \\
\hline & \multicolumn{5}{|c|}{ (In meticais per kilogram) } \\
\hline \multicolumn{6}{|l|}{ Cottonseed } \\
\hline Grade I & 3,300 & 2,950 & 2,300 & 2,500 & 2,700 \\
\hline Grade II & 3,100 & $2,60 n$ & 1,950 & 2,100 & 2,100 \\
\hline Peanuts $3 /$ & 3,774 & 5,017 & 4,547 & 4,882 & ... \\
\hline Whole rice 3 & $\ldots$ & $\ldots$ & $\ldots$ & $\ldots$ & $\ldots$ \\
\hline Cashow nuts 4 ' & 4,000 & 3,850 & 3,726 & 3,680 & $\ldots$ \\
\hline Copra 4i & 1,874 & 1,848 & 1,789 & 1,671 & $\cdots$ \\
\hline \multicolumn{6}{|l|}{ Beans 4/ } \\
\hline Type I & $\ldots$ & $\ldots$ & $\ldots$ & $\ldots$ & $\cdots$ \\
\hline Type Il & $\ldots$ & $\ldots$ & $\ldots$ & $\ldots$ & $\ldots$ \\
\hline White maize $3 f$ & 1,388 & 1,503 & $t, 435$ & 1,467 & $\ldots$ \\
\hline Tobacco $3 / 4 /$ & 13,365 & 13,182 & 12,757 & 11,920 & $\ldots$ \\
\hline & \multicolumn{5}{|c|}{ (Ansual percentage change) } \\
\hline \multicolumn{6}{|l|}{ Cottonseed } \\
\hline Grade 1 & -15.4 & -10.6 & -22.0 & 8.7 & 8.0 \\
\hline Grade II & 3.3 & -16.1 & -25.0 & 7.7 & 0.0 \\
\hline Peanuts & 2.1 & 32.9 & .9 .4 & 7.4 & $\ldots$ \\
\hline Whole rice & $\ldots$ & $\ldots$ & $\cdots$ & $\ldots$ & $\ldots$ \\
\hline Cashew nuts & 16.7 & -3.8 & -3.2 & -1.2 & $\ldots$ \\
\hline Copm & 10.2 & -1.4 & .32 & -6.6 & $\ldots$ \\
\hline \multicolumn{6}{|l|}{ Beans } \\
\hline Type I & $\cdots$ & $\cdots$ & $\cdots$ & $\cdots$ & ... \\
\hline Type II & $\cdots$ & $\ldots$ & $\ldots$ & $\cdots$ & $\cdots$ \\
\hline White tnaize & -18.5 & 8.3 & -4.5 & 2.2 & $\ldots$ \\
\hline Tobacco & 10.2 & -1.4 & $-3,2$ & -6.6 & s. \\
\hline \multicolumn{6}{|l|}{ Memorandum item: } \\
\hline Exchange rate (meticais per U.S. dollar; period average) & 11,546 & 11,850 & 12,689 & 15,689 & 20,707 \\
\hline
\end{tabular}

Source: Mozambican authorities.

1/ All minimum agricultural prices beçame indicative prices in 1996/97.

2/ Prices are set in the fall before the planting season.

3/ Prices were liberalized in $1996 / 97$.

4/ Frices were liberalized in 1997/98. 
Table 13. Mozanbique Pioes Admunistered by He Government, 1999-2002

\begin{tabular}{|c|c|c|c|c|c|c|c|c|c|c|c|c|c|c|c|c|c|}
\hline & $\begin{array}{l}1999 \\
\text { Jar1. }\end{array}$ & $\begin{array}{l}1999 \\
\text { Apsil }\end{array}$ & $\begin{array}{l}1999 \\
\text { May }\end{array}$ & $\begin{array}{l}1999 \\
\text { Junc }\end{array}$ & $\begin{array}{r}200 \% \\
\text { March }\end{array}$ & $\begin{array}{l}20000 \\
\text { Aprri }\end{array}$ & $\begin{array}{l}7000 \\
\text { May }\end{array}$ & $\begin{array}{l}2000 \\
\text { Aug }\end{array}$ & $\begin{array}{l}2500 \\
\text { Sepp }\end{array}$ & $\begin{array}{l}200 ! \\
\text { April' }\end{array}$ & $\begin{array}{c}2001 \\
\text { July }\end{array}$ & $\begin{array}{l}2001 \\
\text { Aur. }\end{array}$ & $\begin{array}{l}2001 \\
\text { Sep. }\end{array}$ & $\begin{array}{l}2001 \\
\text { Nov. }\end{array}$ & $\begin{array}{c}2002 \\
\text { Feb. }\end{array}$ & $\begin{array}{r}2002 \\
\text { Marect }\end{array}$ & $\begin{array}{l}2002 \\
\text { April }\end{array}$ \\
\hline \multicolumn{18}{|l|}{ City or Mizpoto } \\
\hline & \multicolumn{17}{|c|}{ (In met ticais per uni) } \\
\hline Wattes & 3,375 & 4,050 & 4050 & 4,050 & 4,050 & 4,050 & 4,050 & 1050 & 4,050 & 4,050 & 5,170 & 5,170 & 5,170 & $5,1 \pi$ & 5,170 & 5,170 & 5,170 \\
\hline Electricily & 1,40 & 1,430 & 1,430 & 1,430 & 1,430 & 1,430 & 1,430 & 1,593 & 1,593 & 1,593 & i, 593 & 1,593 & 1,984 & 2,196 & 2,351 & 2,351 & 2,351 \\
\hline Passenger taricab scrvie: & 2,000 & 2,000 & 2,000 & 2,000 & 2,500 & 3,000 & 3,000 & 3,000 & 3,000 & 3,000 & 3,000 & 3,000 & 3,000 & 8,000 & 4,000 & 4,806 & 5,000 \\
\hline Lotal tekphone servios & 950 & 1,025 & 1,025 & 1,000 & 1,000 & 1,000 & 1,000 & 1,000 & 1,410 & $1,00,3$ & 1,603 & 1,603 & 1,603 & 1,603 & 2,106 & 2,106 & 2,106 \\
\hline National telephone service & 4,750 & 5,125 & $\$, 125$ & 5,000 & 5,000 & $s, 000$ & 5,000 & 5,000 & 5,640 & 6,412 & 6,412 & 6,412 & 6,417 & 6,412 & 5,494 & 5,494 & 5,494 \\
\hline \multirow{2}{*}{ Interrationni teleghone service } & 18,163 & 17,795 & 17,795 & 17,793 & 17,993 & 17,793 & 17.793 & 17,793 & 97,763 & 17,108 & 17,108 & $17,10 \mathrm{~B}$ & 17,108 & 17,108 & 19,244 & $19,24 d$ & 19,244 \\
\hline & \multicolumn{17}{|c|}{ (Percent changes) } \\
\hline Water & $\ldots$ & 20.0 & 0.0 & 0,0 & 80 & 0,0 & 0.0 & 0.0 & 0.0 & 0.0 & 27.7 & 0.0 & 0.0 & 00 & 0.0 & 0.0 & 0.0 \\
\hline Eectricity & $\ldots$ & 0.0 & 0.0 & 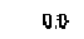 & 0.0 & 00 & 0.0 & 11.4 & oft & 0.0 & 0.0 & 0.0 & 245 & 10.7 & 7.1 & 0.0 & 0.0 \\
\hline Pessanger taxicab sarvice & $\ldots$ & 0.0 & 0.0 & 0.0 & 25.0 & 200 & 0.0 & 0.0 & 0.0 & 0.0 & 0.0 & 0.0 & Do & 33.3 & 0.0 & 20.0 & 4.2 \\
\hline Local telephore servitz & $\ldots$ & 7.9 & 0.0 & -2.4 & 0.0 & 0.0 & 0.0 & 0.0 & 410 & 13.7 & on & 0.0 & 0.0 & 00 & 31.4 & 0.0 & 0.0 \\
\hline National telephone service & $\ldots$ & 79 & 0,0 & -2.4 & 0.0 & 0.0 & 0.0 & 00 & 12.8 & 13.1 & 0.0 & 0.0 & 0.0 & 0.0 & .14 .3 & 0.0 & 0.0 \\
\hline Intenational telephone setvice & $\ldots$ & .1 .0 & 0.0 & 0.0 & 0.0 & 0.0 & 0.0 & 0.0 & 00 & -3.8 & no & 00 & 0.0 & 0.0 & 123 & on & 0,0 \\
\hline \multicolumn{18}{|l|}{ City of Bein } \\
\hline & \multicolumn{17}{|c|}{ (In meticais per unit) } \\
\hline$W_{\Delta t}$ & 2,700 & 3,220 & 3,230 & 3,220 & 3,228 & 3,220 & 3.220 & 3.220 & 3220 & 3,220 & 4,354 & 4,354 & 4,354 & 4,354 & 4,354 & 4,354 & 4,394 \\
\hline Electricity & 1,430 & 1,430 & 1,430 & 1,430 & 1,439 & 1,430 & 1,430 & 1.593 & 1,593 & L.593 & 1,593 & 1,993 & 1,984 & 2,196 & 2,351 & 2,351 & 2,351 \\
\hline Passenger laxticab service & 2,000 & 2,000 & 3,000 & 2,000 & 2,000 & 2,000 & 2,000 & 3,000 & 3,000 & 3,000 & 3,000 & 3,000 & 3,000 & 3,000 & 3,000 & 3,000 & 3,000 \\
\hline 3ocal lelephone scrice & 1,000 & 1,030 & 1,025 & 1,000 & 1,000 & 1.000 & 1,000 & 1,000 & 1.410 & 1,603 & 1.603 & $1,6 \mathrm{GP}$ & 1,603 & 1,603 & 2,106 & 2,106 & 2,106 \\
\hline Nationas telephorte service & 4,750 & 5,125 & 5,125 & 5,000 & 5,000 & 5,000 & 5,000 & 5,002 & 9,649 & 6,417 & $6,4: 2$ & 0,112 & 8,412 & 6,412 & 9,494 & 5,494 & 3,484 \\
\hline \multirow[t]{2}{*}{ International talephone service } & 98,163 & 17.795 & 17,799 & 17,793 & 17,793 & 17,793 & 17.793 & 37,793 & 17,793 & 17.108 & 17,108 & 27,108 & 17,100 & 17,108 & 19,244 & $\$ 9,244$ & $19,20 \mathrm{~d}$ \\
\hline & \multicolumn{17}{|c|}{ (Ptrcen! changes) } \\
\hline WateT & ... & 19.3 & 0.0 & 0.0 & 0.0 & 0.0 & 0.0 & 0.0 & 0.0 & 0.0 & 35.2 & 00 & 0.0 & 0.0 & 0.0 & 0.0 & 0.0 \\
\hline Electricity & - & 0.0 & 0.0 & 0.0 & 0.0 & 0.0 & 0.0 & 11,4 & 0.0 & 0.0 & 0.0 & 0.0 & 24.5 & 10.7 & 7.1 & 0.0 & a. \\
\hline 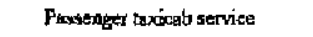 & . & 0.0 & 0.0 & 0.0 & 0.0 & 0.0 & 0.0 & 90.0 & 0.0 & 0.0 & 0.0 & a. & 0.0 & ao & 0.0 & 0.0 & 0.0 \\
\hline Local telephone service & $\ldots$ & 50 & -2.4 & -2.4 & 0.0 & 0.0 & 0.0 & 0.0 & 41.0 & 13.7 & 0.0 & 0.0 & 00 & 0.0 & 31.4 & 0.0 & 0.0 \\
\hline Intional talephonts service & ... & 7.8 & 0.0 & .2 .4 & 0.0 & 0.0 & 0.0 & 0,0 & 22.8 & 13.7 & 0.0 & D.0 & 0.0 & 0.0 & .143 & 0.0 & 6.0 \\
\hline Internationial telephane servace & & -2.0 & 8.0 & 0.0 & 0.0 & 0.0 & 0.0 & 0.0 & 0.0 & -3.8 & 0.0 & 0.0 & 0.0 & 0.0 & 13.5 & 0.0 & 0.0 \\
\hline \multicolumn{18}{|l|}{ City or Nannpula } \\
\hline & \multicolumn{17}{|c|}{ (th metiogis per urit) } \\
\hline Water & 3,160 & 2,740 & 2,740 & $2,74 B$ & 2,740 & 2.740 & 2,740 & 2,740 & 2,740 & 2,740 & 2,740 & 3,104 & 3,422 & 3,422 & $3,+2$ & 3,422 & 3,422 \\
\hline Electricity & 1,430 & 1,430 & 1.430 & $\mathrm{I}, 430$ & 1,430 & 1.430 & 1,430 & 1,593 & $i, 593$ & $1, \$ 93$ & 1,593 & 1,593 & 1,984 & 2,196 & 2,351 & 2,391 & 2,351 \\
\hline Passeriget twacab service & 3,000 & 5,000 & 5,000 & 9,090 & 5,000 & 5,000 & 7,000 & 7.000 & 7.000 & 7,000 & 3,000 & 7,000 & 7.080 & 7.000 & 7,000 & 7,000 & 7,000 \\
\hline \multirow[t]{2}{*}{ Local tctephone service } & 950 & 1.025 & 1,000 & 1,000 & 1,000 & 1,000 & 1,000 & 1,000 & 1,410 & 1,603 & 1.603 & $1.06,3$ & 1,6033 & 1,603 & 2,106 & 2,106 & 2,106 \\
\hline & \multicolumn{17}{|c|}{ (Perant ehanger) } \\
\hline Watre & ... & 269 & 0.0 & $\Delta 0$ & 0.0 & 0.0 & 00 & 0.0 & 0.0 & 0.0 & 0.0 & 13.3 & 10.1 & 0.0 & 0.8 & 0.0 & 0.0 \\
\hline Eleetricily & $\ldots$ & 0.0 & 0.0 & 0.0 & 0.0 & $a . a$ & 0.8 & $\$ 1.4$ & 0.2 & 0.9 & 0.0 & 00 & 24.5 & 10.7 & T.. & 0.2 & 0.0 \\
\hline Tassenger taxicab service & $\ldots$ & 0.0 & 0.0 & 0.0 & 0.0 & 0.0 & 40.0 & 0.0 & 0.0 & 0.0 & 0.0 & 00 & 00 & 0.0 & 0.0 & 0.0 & 0.0 \\
\hline Local telephathe service & $\ldots$ & 1.9 & .2 .4 & 0.0 & 0.0 & 0.0 & D. 0 & 0.0 & 41.0 & 13.7 & 0.0 & 0,0 & 0.0 & AD & 31.4 & 00 & 00 \\
\hline
\end{tabular}

Sowse: Morembican authosities.

CInternational Monetary Fund. Not for Redistribution 


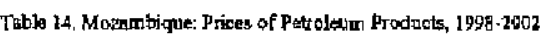

\begin{tabular}{|c|c|c|c|c|c|c|c|c|c|c|c|c|c|c|c|c|c|c|c|c|c|}
\hline & $\begin{array}{r}1998 \\
\text { Metrch }\end{array}$ & $\begin{array}{l}1998 \\
\text { April }\end{array}$ & $\begin{array}{l}1998 \\
\text { Dec. }\end{array}$ & $\begin{array}{c}1999 \\
19 \mathrm{ar}\end{array}$ & $\begin{array}{l}1999 \\
\text { Sep. }\end{array}$ & $\begin{array}{l}1999 \\
\text { Oct. }\end{array}$ & $\begin{array}{l}20000 \\
\text { Feth. }\end{array}$ & $\begin{array}{r}2000 \\
\text { Masch }\end{array}$ & $\begin{array}{l}2000 \\
\text { Myy }\end{array}$ & $\begin{array}{l}2000 \\
\text { July }\end{array}$ & $\begin{array}{l}2000 \\
\text { Oct. }\end{array}$ & $\begin{array}{l}2001 \\
\text { Feb. }\end{array}$ & $\begin{array}{r}2001 \\
\text { Marey }\end{array}$ & $\begin{array}{l}2001 \\
\text { May }\end{array}$ & $\begin{array}{l}200: 1 \\
\text { lanne }\end{array}$ & $\begin{array}{l}2001 \\
\text { July }\end{array}$ & $\begin{array}{l}2001 \\
\text { sep. }\end{array}$ & $\begin{array}{l}2001 \\
\text { Now. }\end{array}$ & $\begin{array}{c}2007 \\
\text { Jant. }\end{array}$ & $\begin{array}{r}2007 \\
\text { March. }\end{array}$ & $\begin{array}{l}2001 \\
\text { May }\end{array}$ \\
\hline & \multicolumn{21}{|c|}{ (In setickis per Fter, tuless otherwike indicated) } \\
\hline Butane (meticais pa kelogiarn) & 6,662 & 6,615 & 6,664 & 5,652 & 7,018 & 7,267 & 7,158 & 8,268 & 8,720 & 8,720 & 9,050 & 10,310 & 10,533 & 11,278 & 12,539 & $1,3,15 d$ & 12,293 & 13,234 & $13, ₫ \$ 4$ & 34,169 & 13,979 \\
\hline Diesel & 4,750 & $\$, 750$ & $4,44 a$ & 4,340 & 4,340 & 4,340 & 5,710 & 6,250 & 7,000 & 7,110 & 8,850 & 3,080 & 7.440 & 8,\%a & 8,370 & 5,400 & $\$, 840$ & $\$, 340$ & 8,230 & 9,244 & 9,280 \\
\hline Fuei cil $\mathbf{l} /$ & 2,489 & $2, \mathbf{4 8 0}$ & 7,638 & 2,567 & 2,871 & 3,485 & 3,652 & 4,311 & 4,549 & 4,453 & 4,638 & $s, 391$ & 5,160 & 5,699 & 5, , 87 & $6.10 \hat{8}$ & 6,279 & 6,470 & 5,399 & 6,087 & 5,941 \\
\hline Ousoline & 6,500 & 6,369 & 6,350 & 6,190 & 6,310 & 6,620 & 3,580 & 8,040 & 9,320 & 9,850 & 9,165 & 8,800 & 9,330 & 10,910 & 11,320 & 11.323 & 9,500 & 9,160 & $\$ 860$ & 11,212 & 11,310 \\
\hline Jet Al & 2,688 & 2,831 & 2,879 & 2,556 & 3,783 & 3,231 & 3,844 & 4,613 & 5,071 & $5,3+4$ & 6.333 & 4,644 & 5.530 & 6,255 & 6,634 & 7,222 & 6,766 & 6,260 & 6,046 & 6.461 & 6,775 \\
\hline Kerosene & 2,250 & 2,450 & $2,4+2$ & 2,120 & 2,350 & 2,350 & 2.780 & 3,330 & 3990 & 4,770 & 5,900 & 4,210 & 5,040 & 5,200 & 5,200 & 6,769 & 6,330 & 5,840 & 5,620 & 6,041 & 6.350 \\
\hline \multicolumn{22}{|l|}{$\begin{array}{l}\text { Memorandum ittus: } \\
\text { Represertstive axchurage rate (meticai }\end{array}$} \\
\hline $\begin{array}{l}\text { Representustive exchunge rate (metioai } \\
\text { dollar; end of period) } 2 /\end{array}$ & 11,594 & 11,575 & 12,366 & 12,194 & 12,933 & 13,000 & 14,977 & 15,407 & 16,043 & 16,194 & 16,319 & 18,231 & 18,657 & 20,608 & 7,933 & 21,788 & 22,182 & 23,080 & 23,462 & 27,623 & $\ldots$ \\
\hline Diest (U.S. dollars per U. S. gallst) & 1.6 & 1.6 & 1.4 & 13 & 1.3 & 1.3 & 1.4 & 1.5 & 1.7 & 17 & 2.1 & 1.5 & 1.5 & 1.6 & 13 & 1.7 & i.s & 1.5 & 1.3 & 1.3 & ... \\
\hline \multirow[t]{2}{*}{ Casuiline (U.5. dollums per U.S. galion) } & 2.1 & 2.1 & 20 & 19 & 1.8 & 1.9 & 1.7 & 2.0 & 2.2 & 2.2 & 2.2 & 1.8 & 5.9 & 2.0 & 20 & 2.0 & 1.6 & 1.5 & 1.4 & 1.8 & , \\
\hline & \multicolumn{21}{|c|}{ (Pertentage changes, unless ollicewise indicated) } \\
\hline Bulfme (meticais pef lillogram) & -1.5 & -0.7 & 0.7 & .152 & 24.2 & 2.7 & .0 .7 & 15.5 & 5.5 & 0.0 & 3.8 & 13.9 & 2.2 & 5.1 & 32 & 49 & .65 & 7.7 & 2.0 & 3.0 & .1 .3 \\
\hline Dieset & $-\$, 4$ & 0.0 & -6.5 & -2.3 & 0.0 & 0.0 & 20.0 & 20.0 & 120 & 1.6 & 24.5 & .20 .0 & 5.1 & 15.7 & 2.2 & 6.7 & -5.5 & 4.5 & .11 .9 & 12,3 & 4.3 \\
\hline Futel oul if & -3.4 & -0.4 & 6.4 & -2.7 & 11.8 & 21.4 & 9.9 & 16.8 & 5.5 & .2 .1 & 4.2 & 16.2 & -4.3 & 10.4 & $-0: 2$ & 7.4 & 2.8 & 3.0 & -16.5 & 12.7 & -2.4 \\
\hline Gasoline & -3.0 & -2.2 & -0.2 & -2.5 & 1.9 & 49 & 14.5 & 6.1 & 15,9 & 5.7 & $-7,0$ & 4.8 & 6.0 & 16.9 & 3.8 & 0.0 & -16.1 & -3.6 & -33 & 26.5 & 0.9 \\
\hline Iet $N$ & 19.9 & 7.2 & -0.1 & .11 .2 & 9.8 & 16.0 & 19.0 & 20.0 & 9.9 & 4.6 & 19.4 & .26 .7 & 19.7 & 13.1 & 6.1 & 89 & -6.3 & -7.5 & -3.4 & 6.8 & 4.9 \\
\hline Ketosence & -23.9 & 8.9 & 0.2 & -6.3 .3 & 10.8 & 0.0 & 18.3 & 19.8 & 19.3 & 19.5 & 237 & .78 .8 & 19.7 & 17.4 & 5.1 & 5.0 & 6.4 & -7.7 & -3.8 & 7.5 & 5.1 \\
\hline \multicolumn{22}{|l|}{ Menoranderan ittems: } \\
\hline Rapresentutive axclangeg rats (nedieais & & & & & & & & & & & & & & & & & & & & & \\
\hline dollar; end of period $z^{2}$ & 02 & -0.5 & 6.8 & 0.2 & 4.3 & 0.5 & 113 & 7.1 & 3.5 & 0.9 & 0.8 & 11.7 & 24 & 10.4 & 6.A & 0,9 & 1.8 & 4.0 & 1.4 & 0.9 & .. \\
\hline Diesel (U.S. dollars fer U.S. gallon) & -9.5 & -22.7 & .12 .5 & -2.5 & .4 .2 & -0.5 & 74 & 12,0 & B. 2 & 0.6 & 23.5 & .28 .4 & 2.6 & 5.7 & -4.0 & 7.4 & -7.2 & 0.4 & -13.1 & 11.3 & ... \\
\hline 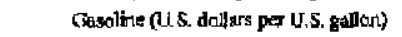 & -7.3 & -4.6 & -8.0 & -4.9 & -2.3 & 4,4 & -10.2 & 13.4 & 12.0 & $-0,9$ & 0.8 & .15 .5 & 3.5 & 5.9 & .25 & 0.7 & -17.6 & .7 .3 & .46 & 75.4 & ... \\
\hline
\end{tabular}

Source; Mozambiasan wuthon'tias; and staff escimatas.

I/ Wholesale pritc.

If The market rate is nosed.

CInternational Monetary Fund. Not for Redistribution 
Table 15. Mozambique: Import Prices of Qi] Products, 1997-2001 1/

\begin{tabular}{|c|c|c|c|c|c|}
\hline Products & 1997 & 1998 & 1999 & 2000 & 2001 \\
\hline & \multicolumn{5}{|c|}{ (U.S. dollars per unit indicated) } \\
\hline Liquefied petroleum gas (butane) (ton) & 240.8 & 227.2 & 232.9 & 222.9 & 230.4 \\
\hline Aviation gasoline (cubic meter) & 178.8 & 266.3 & 266.6 & $\ldots$ & $\ldots$ \\
\hline Premuium gasoline (cubic meter) & 157.1 & 116.9 & 185.8 & 219.3 & 171.8 \\
\hline Jet A1 (cubic meter) & 190.4 & 107.6 & 163.0 & 239.4 & 185.8 \\
\hline Diesel (cubic meter) & 153.4 & 102.4 & 143.4 & 248.6 & 176.5 \\
\hline Fuel oil (cubic meter) & 125.8 & 82.2 & 105.3 & 144.1 & 141.9 \\
\hline Memorandum items: & \multicolumn{5}{|c|}{ (Ratio of import to international prices) $2 /$} \\
\hline Premium gasoline & 1.01 & 1.06 & 1.36 & 0.97 & 0.91 \\
\hline Jet fuel/kcrosene & 1.23 & 1.00 & 1.18 & 1.03 & 0.96 \\
\hline Diesel & 1.04 & 1.01 & 1.14 & 1.15 & 0.96 \\
\hline Fuel oil & 1.37 & 1.28 & 1.16 & 1.07 & 1.27 \\
\hline
\end{tabular}

Sources: Petromoc; International Energy Agency, Intemational Energy Agency Monthly Oil Market Report; and Ministry of Natural Resources and Energy, National Directorate for Energy.

1/ Annual average prices.

2 International prices refer to the Rotterdam market. 
Table 16. Mozambique; Price Structure of Petroleum Products, November 200 |

\begin{tabular}{|c|c|c|c|c|c|c|}
\hline & Butane 1/ & Diesel & Fuel Oil & Gasoline & Jet $A]$ & Kerosene \\
\hline & \multicolumn{6}{|c|}{ (Meticais per liter) } \\
\hline $\operatorname{Cos} 1$ (c.i.f) & $5,653.6$ & $4,252.7$ & $3,391.3$ & $2,992.6$ & $3,783.8$ & $3,783.8$ \\
\hline Customs duties & 282.7 & 212.6 & 169.6 & 149.6 & 189.2 & 189.2 \\
\hline Import charges & 393.0 & 239.5 & 234.7 & 239.5 & 239.5 & 239.5 \\
\hline Importer/ distributor's margin & $3,612.1$ & $1,297.6$ & $1,297.6$ & $1,397.6$ & $1,297.6$ & $1,297.6$ \\
\hline Value-added tax & 1690.0 & 1020.4 & 865.8 & 795.5 & 0.0 & 0.0 \\
\hline Petroleam tax & 284.0 & $1,825.0$ & 328.3 & $3,307.8$ & 430.2 & 0.0 \\
\hline Wholsale price, net 2 ! & $12,142.4$ & $8,966.4$ & $6,407.0$ & $8,786.4$ & $5,940.3$ & $5,520.7$ \\
\hline Transport differential & 148.3 & 53.8 & 53.8 & 53.8 & 53.8 & 53.8 \\
\hline Vatue-added tax & 25.2 & 9.1 & 9.1 & 9,1 & 0.0 & 0.0 \\
\hline Wholsale price, gross & $12,315.9$ & $9,029.4$ & $6,470.0$ & $8,849.3$ & $5,994.1$ & $5,574.5$ \\
\hline Retail's margin & 784.7 & 265.5 & 0.0 & 265.5 & 265.5 & 265.5 \\
\hline Value-added tax & 133.4 & 45.1 & 0.0 & 45.1 & 0.0 & 0.0 \\
\hline Calculated retail price, gross & $13,234.0$ & $9,340,\{\}$ & $6,470.0$ & $9,160.0$ & $6,259.6$ & $5,840.0$ \\
\hline \multicolumn{7}{|l|}{ Memorandum jtems: } \\
\hline Total taxes & $2,415.3$ & $3,112.3$ & $1,372.9$ & $4,307.2$ & 619.4 & 189.2 \\
\hline Total margins & $4,396.8$ & $1,563,1$ & $1,297.6$ & $1,563.1$ & $1,563.1$ & $1,563.1$ \\
\hline \multirow[t]{2}{*}{ Retail price in US\$ per liter $3 /$} & 0.58 & 0.41 & 0.29 & 0.40 & 0.28 & 0.26 \\
\hline & \multicolumn{6}{|c|}{ (In percent of retail price) } \\
\hline \multicolumn{7}{|l|}{ Irrport cost } \\
\hline Margins and taxes & 50.1 & 48.8 & 40.8 & 62.2 & 32.3 & 27.4 \\
\hline Wholesale price, net & 91.8 & 96.0 & 99.0 & 95.9 & 94.9 & 94.5 \\
\hline Retail price & 100.0 & 100.0 & 100.0 & 100.0 & 100.0 & 100.0 \\
\hline \multicolumn{7}{|l|}{ Memorandum items: } \\
\hline Total taxes & 18.3 & 33.3 & 21.2 & 47.0 & 9.9 & 3.2 \\
\hline Total margins & 33.2 & 16.7 & 20.1 & 17.1 & 25.0 & 26.8 \\
\hline Total taxes and margins & 51.5 & 50.1 & 41.3 & 64.1 & 34.9 & 30.0 \\
\hline
\end{tabular}

Source: Mozambican authorities; and Fund staff estimates.

1) Meticais per kilogram.

2/ Includes some residual costs.

3) In the case of butane, U.S. dollars per kilogram. 
Table 17. Mozanhique: Minimum Monthly Wage, 1997-2002 I/

\begin{tabular}{|c|c|c|c|c|c|c|}
\hline & $\begin{array}{l}1997 \\
\text { April }\end{array}$ & $\begin{array}{l}1998 \\
\text { April }\end{array}$ & $\begin{array}{l}1999 \\
\text { April }\end{array}$ & $\begin{array}{r}2000 \\
\text { July }\end{array}$ & $\begin{array}{l}2001 \\
\text { April }\end{array}$ & $\begin{array}{l}2002 \\
\text { April }\end{array}$ \\
\hline & \multicolumn{6}{|c|}{ (In units indicated) } \\
\hline \multicolumn{7}{|l|}{ Nominal monthly wage (in meticais) } \\
\hline Agricultural worker 2 & 209,960 & 238,873 & $\cdots$ & $\cdots$ & 459,270 & 560,309 \\
\hline Nonagricultural worker & 311,794 & 353.886 & 450,000 & 568,980 & 665,707 & 812,163 \\
\hline Technicaliadninistrative worker & 311,394 & 353,886 & 450,000 & 568,980 & 655,707 & 812,163 \\
\hline \multicolumn{7}{|l|}{ Real wage index (April 1998=100) } \\
\hline Agriculntral worker & 90 & 100 & $\cdots$ & $\ldots$ & 173 & 173 \\
\hline Nonagricultural worker & 9] & 100 & 124 & 139 & 165 & 165 \\
\hline TechnicaVadministrative worker & 91 & 100 & 110 & 139 & 190 & 190 \\
\hline \multicolumn{7}{|l|}{ Nominal monthly wage (in U.S. dollars) } \\
\hline Agricultural worker & 18 & 21 & $\ldots$ & $\ldots$ & 23 & 24 \\
\hline Nonagricultural worker & 27 & 31 & 36 & 35 & 34 & 34 \\
\hline Technical/adminictrative worker & 27 & 31 & 36 & 35 & 34 & 34 \\
\hline \multicolumn{7}{|l|}{ Memorandum items: } \\
\hline Consumer price index (CPI) (Dec. 1998=100) & 99 & 102 & 105 & 119 & 119 & 145 \\
\hline \multirow[t]{2}{*}{ Exchange rate (meticais per U.S. dollar, end of petiod) } & 11,609 & 11,530 & 12,424 & 16,194 & 19,629 & 23,581 \\
\hline & \multicolumn{6}{|c|}{ (Percentage change) } \\
\hline \multicolumn{7}{|l|}{ Nominal monthly wage (in melicuis) } \\
\hline Agricultural worker & 16.6 & 13.8 & $\cdots$ & $\ldots$ & $\ldots$ & 22.0 \\
\hline Nonagricultural worker & 15.0 & 13.5 & 27.2 & 26.4 & 17.0 & 22.0 \\
\hline Tecturical/adninistrative worker & 15.0 & 13.5 & 27.2 & 26.4 & {$[7.0$} & 22.0 \\
\hline \multicolumn{7}{|l|}{ Real wage index } \\
\hline Agricultural worker & 8.3 & 10.6 & $\ldots$ & $\cdots$ & $\cdots$ & -0.1 \\
\hline Nonagrieultural worker & 5.8 & 10.3 & 24.1 & 11.7 & 18.9 & -0.1 \\
\hline Technical'administrative worker & 6.8 & 10.3 & 10.3 & 25.6 & 36.9 & -0.1 \\
\hline \multicolumn{7}{|l|}{ Nominal monthly wage (is U.S. dollars) } \\
\hline Agricultural worker & 14.2 & 14.6 & $\ldots$ & $\cdots$ & $\cdots$ & 1.6 \\
\hline Nonagricultural worker & 12.6 & 14.8 & 16.8 & -3.0 & -3.5 & 1.6 \\
\hline Technical/administrative worker & 12.6 & 14.8 & 16.8 & -3.0 & -3.5 & 1.6 \\
\hline \multicolumn{7}{|l|}{ Mernorandum jtems: } \\
\hline CPI & 7.6 & 3.0 & 2.9 & 13.3 & 0.0 & 22.1 \\
\hline Exchange rate (meticais per U.S. dollar) & 2.2 & -0.7 & 7.8 & 30.3 & 21.2 & 20.1 \\
\hline
\end{tabular}

Source: Ministry of Platuing and Finance; and staff estimates.

1/ Months stown reter to time of change.

2/ The minimum wage increase for the year 2002 assumes equal increases for agricultural and nonagncultura] workers. 
Table 18. Mozambique: Expenditure on the Social Sectors as defined in the PARPA, 1999-2001 //

(In billions of meticais, uniess otherwise indicated)

\begin{tabular}{|c|c|c|c|}
\hline & 1999 & 2000 & 2001 \\
\hline Total expenditure (excluding bank restructuring costs and interest payments) & 12,491 & 15,663 & 21,158 \\
\hline Total actuaibudgeted expenditure in PARPA priority sectors & 6,895 & 10,798 & 14,006 \\
\hline Education & 1,795 & 3,157 & 4,890 \\
\hline Primaty & 1,410 & 2,744 & 3,891 \\
\hline Postprimary & 384 & 414 & 999 \\
\hline Health & 1,493 & 2,038 & 2,235 \\
\hline HIV/AIDS & 0 & 6 & 132 \\
\hline Infrastructure development & 1,481 & 2,490 & 3,621 \\
\hline Roads & $\ldots$ & $\ldots$ & 1,845 \\
\hline Sanitation and public works & $\ldots$ & $\ldots$ & 1,776 \\
\hline Agriculture and rural development & 583 & 994 & 730 \\
\hline Governance and judicial system & 991 & 1,244 & 1,704 \\
\hline Security and public order & 722 & 843 & 1,095 \\
\hline Governance & 60 & 142 & 249 \\
\hline Judicial system & 209 & 258 & 359 \\
\hline Other priority areas $2 /$ & $\$ 52$ & 869 & 694 \\
\hline Social actions & 69 & 180 & 150 \\
\hline Labor and employment & 55 & 56 & 79 \\
\hline Mineral resources and energy & 428 & 634 & 455 \\
\hline
\end{tabular}

Total actual/budgeted expendinure in PARPA priority sectors $1 /$

(In percent of gross domestic product)

Education

13.3

18.3

18.8

Primary

3.5

5.4

6.5

Postprimary

2.7

4.7

5.2

0.7

0.7

1.3

Health

2.9

3.5

3.0

HIV/AIDS

0.0

0.0

0.2

Infrastructure development

2.9

4.2

Roads

Senitation and public works

Agriculture and rural development

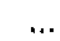

Governance and judicial system

Security and public order

Governance

Judicial system

Other priority areas $2 /$

Social actions 
Table 18. Mozambique: Expenditure on the Social Sectors, 1999-2001 (concluded)

(In percent of total expenditure) $3 /$

Total actual/budgeted expenditure in PARPA priority sectors $1 /$

$\begin{array}{rrr}55.2 & 68.9 & 66.2 \\ 14.4 & 20.2 & 23.1 \\ 11.3 & 17.5 & 18.4 \\ 3.1 & 2.6 & 4.7 \\ 12.0 & 13.0 & 10.6 \\ 0.0 & 0.0 & 0.6 \\ 11.9 & 15.9 & 17.1 \\ \ldots & \ldots & 8.7 \\ \ldots & \ldots & 8.4 \\ 4.7 & 6.3 & 3.5 \\ 7.9 & 7.9 & 8.1 \\ 5.8 & 5.4 & 5.2 \\ 0.5 & 0.9 & 1.2 \\ 1.7 & 1.6 & 1.7 \\ 4.4 & 5.6 & 3.3 \\ 0.6 & 1.1 & 0.8 \\ 0.4 & 0.4 & 0.4 \\ 3.4 & 4.0 & 2.2\end{array}$

Education

Primary

Postprimary

Heakth

HTY/AIDS

Inftastructure development

Roads

Sanitation and public works

Agriculture and rural development

Governance and judicial system

Security and public order

Governance

Judicial systeit

Other priority areas $2 /$

Social actions

Labor and employment

Mineral tesources and energy
列

Memorandun item:

Gross Domestic Product

Source: Ministry of Planning and Finance; and staff estimates.

1/ PARPA stands for National Action Plan for the Reduction of Absolute Poverty, which is the Portuguese acronym for the Poverty Reduction Strategy Paper (PRSP).

2/ Relates to expenditures viewed complementary to PARPA priority areas and which contribute to income generation and employment opportunities.

3/ Excludes bank restructuring costs and interest payments. 
Table 19. Morambique: Number of Households Receiving Food Subsidy Assistance,

March 1997-Decernber 200

\begin{tabular}{|c|c|c|c|c|c|c|c|c|c|c|c|c|c|c|c|c|}
\hline & \multicolumn{4}{|c|}{1997} & \multicolumn{4}{|c|}{1998} & \multicolumn{4}{|c|}{1999} & \multicolumn{2}{|c|}{2000} & \multicolumn{2}{|c|}{2001} \\
\hline & March & June & Sep. & Dec. & Match & June & Step. & Dec. & March & June & Sep. & Dec. & June & Dec. & June & Dec. \\
\hline I'otal househoids & 37,460 & 38,773 & 39,893 & 39,893 & 30,693 & $3 !, 027$ & 33,348 & 35,974 & 38,656 & 38,656 & 39,051 & 39,051 & 40,558 & 48,324 & 57,467 & $66,6.25$ \\
\hline $\begin{array}{l}\text { Eligitle on account of: } \\
\text { malnourished chitdren 1/ } \\
\text { underweight pregnant }\end{array}$ & $3,56,5$ & 4,648 & 5,125 & 5,125 & 3,056 & 2,889 & 3,523 & $.8,830$ & 3,220 & 3,220 & 2,218 & 2,218 & 2,199 & 2,001 & 2,542 & 1,095 \\
\hline womes $y$ & 826 & 826 & 788 & 788 & 395 & 312 & 301 & 367 & 1,546 & 1,546 & 3,533 & 1,533 & 236 & 263 & 173 & 1,306 \\
\hline destitute elderly 3I & 29,587 & 29,587 & 29,182 & 29,182 & 23,791 & 24,295 & 25,479 & 27,412 & $3:, 3: 2$ & 31,312 & 31,486 & 31.486 & $32,95,4$ & 39,695 & 46,979 & 54,624 \\
\hline handicapped 4 & 2,368 & 2,368 & 3.221 & 3,221 & 2,143 & 2,173 & 2,561 & 2,629 & 773 & 772 & 2,566 & 2,566 & 2,911 & 3,481 & 3,708 & 4,121 \\
\hline househoids headed by women s/ & 1,114 & 1,341 & 1,577 & 1,577 & 1,308 & 1,358 & 1,484 & 1,736 & 2,705 & 2,705 & $2,78]$ & $2,78 \mathrm{~L}$ & 1,929 & 1,838 & 2,582 & 2,809 \\
\hline
\end{tabular}

Source: Ministry of Planning and Finance, Office for Vulnerable Population lnitiacjues (GAPVU); tnd National Institute for Social Acrion (INAS).

1/ Malinurished children up to 5 ycars of age.

2) Pregnant women who arc underweight telative to thejr gestation period.

3 Elderly people without means of subsistence living alone or in households with no wage eamers.

4) Seriously handicapred people over 18 yeurs old jiving in poventy and without physical capacities to make a living.

5/ With more than five children, and chronically ill persons. 
Table 20. Mozanbique: Budget Subsidies to Enterprises, 1997 - 2001

(In millions of meticais, unless otherwise specified)

\begin{tabular}{|c|c|c|c|c|c|c|c|c|c|c|}
\hline & \multicolumn{2}{|c|}{1997} & \multicolumn{2}{|c|}{1998} & \multicolumn{2}{|c|}{1999} & \multicolumn{2}{|c|}{2000} & \multicolumn{2}{|c|}{2001} \\
\hline & Est. & $\begin{array}{l}\text { In percent } \\
\text { of total }\end{array}$ & Est. & $\begin{array}{l}\text { In percent } \\
\text { of total }\end{array}$ & Est. & $\begin{array}{c}\text { In percent } \\
\text { of total }\end{array}$ & Est. & $\begin{array}{l}\text { In percent } \\
\text { of total }\end{array}$ & Est. & $\begin{array}{l}\text { In percent } \\
\text { of total }\end{array}$ \\
\hline Agriculture & 11,521 & 29.5 & 6,000 & 12.2 & 7,300 & 15.2 & 3,600 & 6.4 & 3,850 & 5.0 \\
\hline Sugar & 7,616 & 19.5 & 0 & 0.0 & 0 & 0.0 & 0 & 0.0 & 0 & 0.0 \\
\hline Tea and copra & 1,000 & 2.6 & 0 & 0.0 & 0 & 0.0 & 0 & 0.0 & 0 & 0.0 \\
\hline Other enterprises & 2,905 & 7.4 & 6,000 & 12.2 & 7,300 & 15.2 & 3,600 & 6.4 & 3,850 & 5.0 \\
\hline Mineral resources & 2,000 & 5.1 & 7,000 & 14.3 & 1,500 & 3.1 & 1,000 & 1.8 & 1,200 & 1.6 \\
\hline Coal (Carbomoc) & 2,000 & 5.1 & 7,000 & 14.3 & 1,500 & 3.1 & 1,000 & 1.8 & 1,200 & 1.6 \\
\hline Other minerals & 0 & 0.0 & 0 & 0.0 & 0 & 0.0 & 0 & 0.0 & 0 & 0.0 \\
\hline Other industries & 25,500 & 65.3 & 36,000 & 73.5 & 39,100 & 81.6 & 51,905 & 919 & 72,290 & 93.5 \\
\hline Electricity (EDM) & 0 & 0.0 & 0 & 0.0 & 0 & 0.0 & 0 & 0.0 & 0 & 0.0 \\
\hline Construction matcrial & 0 & 0.0 & 0 & 0.0 & 0 & 0.0 & 0 & 0.0 & 0 & 0.0 \\
\hline Othcr enterprises & 3,000 & 7.7 & 7,500 & 15.3 & 4,800 & 10.0 & 6,005 & 10.6 & 12,128 & 15.7 \\
\hline Information services & 22,500 & 57.7 & 28,500 & 58.2 & 34,300 & 71.6 & 45,900 & 81.2 & 60,162 & 77.8 \\
\hline Total subsidies & 39,021 & 100.0 & 49,000 & 100.0 & 47,900 & 100.0 & 56,505 & 100.0 & 77,340 & 1000 \\
\hline \multicolumn{11}{|l|}{ Memorandum item: } \\
\hline $\begin{array}{l}\text { Total subsidies as a percent } \\
\text { of gross domestic product }\end{array}$ & 0.10 & & 0.10 & & 0.09 & & 0.10 & & 0.10 & \\
\hline
\end{tabular}

Source: Ministry of Planning and Finarice. 
(In billions of meticais)

\begin{tabular}{|c|c|c|c|c|c|}
\hline & 1997 & 1998 & 1994 & 2000 & 2001 \\
\hline Total revenue & 4,623 & 5,324 & 6,207 & 7,463 & 9,616 \\
\hline Tax revenue & 4,235 & 4,932 & 5,733 & 6,857 & 8,589 \\
\hline Taxes on income and profits & 879 & 963 & 867 & 1,008 & 1,498 \\
\hline Taxes on goods and services & 2,389 & 2,882 & 3,638 & 4,331 & 5,379 \\
\hline Taxes on intemational trasic & 812 & 937 & 1,046 & 1,297 & 1,477 \\
\hline Other taxes & 155 & 150 & 183 & 221 & 235 \\
\hline Nontax revenue & 388 & 392 & 474 & 606 & 1,027 \\
\hline Total expenditure and net lending & 9,498 & $10,1 A 1$ & 12,815 & 16.735 & 23,221 \\
\hline Current experditure & 4,272 & 5,268 & 6,332 & 7,836 & 10,489 \\
\hline Budgel year & 4,361 & 5,259 & 6,347 & 8,077 & 10,653 \\
\hline Carapensation to cmployees & 1,445 & 2,097 & 2,995 & 3,844 & 4,898 \\
\hline Wages and salaries & $\ldots$ & 1,873 & 2,806 & 3,593 & 4,541 \\
\hline Other & $\ldots$ & 224 & 189 & 251 & 357 \\
\hline Guods and services & 1,334 & 1,544 & 1,646 & 1,976 & 2,400 \\
\hline Incerest on public debt & 530 & 463 & 324 & 118 & 478 \\
\hline Domestic & 34 & 21 & 6 & 14 & 328 \\
\hline Excernạd & 496 & 442 & 318 & 104 & 150 \\
\hline Transfer payments & 750 & 874 & 1,085 & 1,664 & 2,230 \\
\hline Local and district governments & 80 & 102 & 105.2 & 224 & 273 \\
\hline Political parties & si & 57 & 66 & 71 & 75 \\
\hline Househoiks & 420 & 463 & 646 & 937 & 1,371 \\
\hline Pensions & 354 & 390 & 559 & 748 & 1,043 \\
\hline Welfare payzuents & 48 & 37 & 87 & 129 & 176 \\
\hline Other & 18 & 36 & 0 & 61 & 153 \\
\hline Subsidies to entenprises & 47 & 46 & 54 & 56 & 69 \\
\hline Abroad & 152 & 206 & 214 & 376 & 443 \\
\hline Finhassica & 132 & 182 & 214 & 340 & 412 \\
\hline Internationai organizations & 20 & 24 & 0 & 36 & 32 \\
\hline Other & 302 & 281 & 297 & 475 & 647 \\
\hline Wet float 1 if & .89 & 9 & -15 & -241 & -164 \\
\hline Curcent balance & 351 & 56 & -125 & -373 & .873 \\
\hline Capital expendinure & 4,816 & 4,575 & 6,001 & 7,826 & 10,428 \\
\hline Budget year & 3,754 & 4,843 & 5,734 & 8,177 & 10,516 \\
\hline Extemal project gants & 1,562 & 2,091 & 2,710 & 3,456 & 5,625 \\
\hline External project joans & 1,435 & 1,673 & 1,306 & 1,602 & 1,101 \\
\hline Locally financesd & 742 & 1,079 & 1,717 & 2,688 & 3,296 \\
\hline Doner-fimenced outside budget & 15 & 0 & 0 & 0 & 0 \\
\hline HJPC iníliative & 0 & 0 & 0 & 0 & 0 \\
\hline Net float & 1,062 & -268 & 267 & -351 & -88 \\
\hline Net lending & 410 & 298 & 482 & 1,073 & 2,304 \\
\hline Unallocated revenue $(+) / \exp$ esuditure $(t)$ & EI9 & -105 & -220 & 443 & 0 \\
\hline
\end{tabular}


Table 21. Mozanbique; Governtatemt Finances, 1997-2001 (concluded)

(In billions of meticais)

\begin{tabular}{|c|c|c|c|c|c|}
\hline & 1993 & 1998 & 1999 & 2000 & 2001 \\
\hline Overall balance before grants & $-4,736$ & $-4,923$ & $-6,828$ & $-\$, 329$ & $-13,605$ \\
\hline Grants received & 3,705 & 3,818 & 6,073 & 6855 & 9,637 \\
\hline Projezt & 1,962 & 1,894 & 2,787 & 3810 & 5,985 \\
\hline Nonproject & 1,743 & 1,924 & 3,287 & 3045 & 3,652 \\
\hline Overall balance after grants & $-1,031$ & $-1,105$ & .754 & $-1,974$ & $-3,968$ \\
\hline Extennal borrowing (net) & 2,329 & 2,172 & 910 & 1674 & 2917 \\
\hline Disbursements & 2,752 & 2,671 & 1,394 & 1956 & 3591 \\
\hline Project & 1,897 & 5,641 & 1,394 & 1484 & 2359 \\
\hline Nonproject & 855 & 1,030 & 0 & 472 & 1232 \\
\hline Cash anortization & 423 & 499 & 483 & 282 & 674 \\
\hline Dumestic financing (net) & $-1,298$ & $-1,067$ & -117 & 479 & 965 \\
\hline Banking systerd & $-1,298$ & $-1,067$ & -377 & 479 & 965 \\
\hline Other (includimg residual) & 0 & 0 & 60 & 0 & 0 \\
\hline \multicolumn{6}{|l|}{ Mertoramium items; } \\
\hline Primary current batance $1 /$ & 881 & 519 & 199 & -255 & -396 \\
\hline Domestic primary balance before grants $2 /$ & 291 & -289 & $-1,780$ & $-3,304$ & $-5,272$ \\
\hline
\end{tabular}

Sources: Mozambican authorities; and staff estimates.

1/ Budget procedures in Mozambique allow for a 3-[onth complementary period, meaming that each year, from January to March, expenses can be incurred in executing the previous' year budget. Net float corresponds to the expenses incurred in January to March but which relate to the previous year budget, asinus what is left in the budget at the end of the previous year to be paid during next fiseal year.

2) Current revenue minus nominterest current expendilure.

3/ Total revenue mints noninterest current expenditure minus local ty financed capital expenditure and locally financed net lending. 


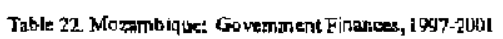

(tin peicent o: GDP)

\begin{tabular}{|c|c|c|c|c|c|}
\hline & 1997 & 1993 & 1949 & 2000 & $200 !$ \\
\hline Total tevenue & $! 1.4$ & 11.4 & 120 & 12.7 & 18.9 \\
\hline Tax reventus & 10.4 & 10.5 & $\mathrm{LLO}$ & 11.6 & 11.5 \\
\hline Taxes on itstune and profits & 2.2 & 2.1 & 1.7 & 1.7 & 2.0 \\
\hline Tiaces on grods and tervites & 5.9 & G. L & 7.0 & 7.4 & 7.2 \\
\hline Tinges on international troce & 20 & 2.0 & 20 & 2.2 & 2.0 \\
\hline Other taxes & 0.4 & 0.3 & 0.4 & 0.4 & 0.3 \\
\hline Nantiñ revenuq & 1.0 & 0.8 & 09 & 1.0 & 1.4 \\
\hline Tasal expendioune and net lendiag. & 23.4 & 21.6 & 24.7 & 28.4 & 31.1 \\
\hline Cutrent expenditure & 10.5 & $\mathrm{i} 1.2$ & 12.2 & 13.3 & 14.0 \\
\hline Budgel year & 10.8 & 11.2 & 12.2 & 19.7 & 1.3 \\
\hline Compenisation ta etrployees & 3.6 & 4.5 & $5 \mathrm{~g}$ & 5.5 & 6.6 \\
\hline Guokls and services. & 3.3 & 33 & 32 & 3.4 & 12 \\
\hline linterexr on puigted debt & 13 & 1.0 & 06 & 0.2 & 0.6 \\
\hline Dannesuic & 0.1 & 0.0 & 0.0 & 0.0 & 0.4 \\
\hline Exuemal & 1.2 & 0.9 & 0.6 & 0.2 & 02 \\
\hline Transfer pay icuents & 1.8 & 1.9 & 2.1 & 2.8 & 3.0 \\
\hline Lorcal and districat govatimarits & 0.2 & 02 & 01 & 0.4 & 0.4 \\
\hline Political parties & 0.1 & 0.1 & 0.1 & 0.1 & 0.1 \\
\hline Jicus etrolds & 1.0 & 1.0 & 1.2 & 1.6 & 1.8 \\
\hline Pensions & 0.9 & 0.8 & 1.1 & 1.3 & 1.4 \\
\hline Welfarr payments & 0.1 & 0.1 & 0.2 & 0.2 & 0.2 \\
\hline Onther & 0.0 & 0.1 & 0.0 & 0.1 & 0.2 \\
\hline Subsindies रo erterprived & 0.1 & 0.1 & 0.1 & 0.1 & 0.1 \\
\hline Abroad & 9.4 & 0.4 & 0.4 & 0.6 & 0.6 \\
\hline Embassios & 03 & 0.4 & 0.4 & 0.6 & 0.6 \\
\hline Intermaticunal ongargreations & 0.0 & 0.1 & 0,0 & 0.1 & 6.13 \\
\hline Dther & 0.7 & 0.6 & 0.6 & 0.8 & 0.9 \\
\hline Net Eloat & -0.2 & 0.0 & 0.0 & -0.4 & -0.2 \\
\hline Current baknes & 0,9 & 0.1 & $-\mathbf{a} \mathbf{z}$ & -0.6 & -1.2 \\
\hline Capinal expentrirure & 11.9 & 9.8 & 11.6 & 13.3 & 14.0 \\
\hline Budget year & 9.3 & 10.3 & 110 & 13.9 & 14.1 \\
\hline Exterial project grants & 3.9 & 49 & 5.2 & 5.9 & 75 \\
\hline External propecs kasss & 3.5 & 3.6 & 2.5 & 1.7 & 1.5 \\
\hline Localigy fir anced & $\$ . *$ & 2.3 & 3.3 & 46 & 4.4 \\
\hline Donar-financed outside budget & 0.0 & 0.0 & 0.0 & 0.0 & 0.0 \\
\hline HrPe in iniative & 0.0 & 0.8 & 0.0 & as & 80 \\
\hline Ňct Doas U & 2.6 & $-0,6$ & 0.5 & 46 & $-0,2$ \\
\hline inet knding & 1.0 & 0.6 & 0.9 & 1.8 & 3.1 \\
\hline Unailocated revenue $(+)$ expead iture $(-)$ & 03 & -9.2 & -0.4 & 0.8 & 0.0 \\
\hline Oyerall balance before gronss & .11 .7 & -10.5 & -13.2 & -15.0 & +18.2 \\
\hline Gants receivad & 9.1 & 8.1 & 11.7 & 11.6 & 129 \\
\hline Projext & 48 & 4.0 & 5.4 & 6.5 & 8.0 \\
\hline Wenproject & 4.3 & 4 & 6.3 & 5.2 & 69 \\
\hline Overdl ba|anee after BTants & -2.5 & -2.4 & -1.5 & -3.4 & -5.3 \\
\hline Exhras/ bormonit g (nct) & 5.7 & 4.5 & 1.8 & 2.8 & 3.9 \\
\hline Disbumerrents & 6.8 & 5.7 & 27 & 3.3 & 4.8 \\
\hline Project & 4.7 & 3.5 & 2.7 & 2.5 & 3.2 \\
\hline Nomprojuct & 2.1 & 2.2 & 0.0 & 0.8 & 1,6 \\
\hline Cash mmontration & 1.0 & 1.1 & 0.9 & 0.5 & 9.9 \\
\hline Donestic funaroing (net) & .3 .2 & -23 & -0.2 & 0.8 & 1.3 \\
\hline Banking systens & -3.2 & -2.3 & $-0,3$ & 0.8 & 1.3 \\
\hline Ohter (isehuding res ldual) & 0.0 & 0.0 & 0.1 & 0.0 & 0.0 \\
\hline
\end{tabular}

Saujces: Mozambisan authoritics; and staff cstimakes.

W budget pracedures in Mozarnbique allow for a 3-month mmpiementary period, meaning that each year, froin fanuary to

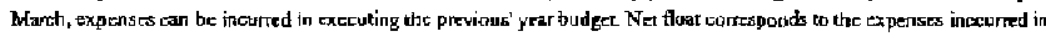
Januagy ro Manch tod which relaie to the previous year budget, minus what is left in the budget at the end of the provious year to be paid dueing next fiscal year.

CInternational Monetary Fund. Not for Redistribution 


\begin{tabular}{|c|c|c|c|c|c|}
\hline & 1997 & 1998 & 1999 & 2000 & 2001 \\
\hline & \multicolumn{5}{|c|}{ (In billions of meticais) } \\
\hline Total revenue & 4,586 & 5,324 & 6,207 & 7,463 & 5,616 \\
\hline Tax revente & 4,235 & 4,932 & 5,733 & 6,857 & 8,589 \\
\hline Taxes an income and profits & 879 & 963 & 867 & $1,00 \%$ & 1,498 \\
\hline Compenies & 388 & 465 & 415 & 372 & 542 \\
\hline Individuals & 491 & 498 & 4.52 & 635 & 957 \\
\hline Taxes an goods and services & 2,385 & 2,882 & 3,638 & 4,331 & 5,379 \\
\hline Turnover tax & 1,330 & 1,559 & 917 & 0 & a \\
\hline Value-added tax & 0 & 0 & 1,397 & 2,917 & 3,782 \\
\hline On domestic production & 0 & 0 & 569 & $1,20 \%$ & 1,629 \\
\hline On imports & 0 & 0 & 828 & 1,709 & 2,153 \\
\hline Cansumpticn taxes & 431 & $\sin$ & 565 & 643 & 773 \\
\hline Tobacco & 31 & 43 & 46 & 91 & 143 \\
\hline Beer and soft drinks & 201 & 278 & 269 & 126 & 378 \\
\hline Other domestic goods & 21 & 29 & 18 & 5 & 1 \\
\hline Imported products & 179 & 222 & 231 & 221 & 250 \\
\hline Tax on petrolem products & $62 \mathrm{~T}$ & 751 & 759 & 771 & 824 \\
\hline Taxes on international trade & $8 \mathrm{t2}$ & 937 & $1,04 \xi$ & 1,297 & 1,477 \\
\hline Other taxes & 155 & 150 & 183 & 221 & 235 \\
\hline Stamp taxes & 71 & 80 & 92 & 112 & 123 \\
\hline Poll taxes & 6 & 4 & 3 & 4 & 5 \\
\hline Other taxes and dulices & 79 & 66 & 88 & 105 & 108 \\
\hline Nantax revenue & 351 & 392 & 474 & 606 & 1,027 \\
\hline Rents frotn real estate & 109 & 185 & 111 & 98 & 113 \\
\hline fees and charges & 36 & A) & 50 & 113 & 65 \\
\hline Social security contributions & 85 & 98 & 112 & 166 & 207 \\
\hline Nonfinancial enterprise profits & 0 & 7 & 0 & 1 & 0 \\
\hline Fighing license fees & 68 & 53 & 60 & 60 & 98 \\
\hline Net privatization revegue & 0 & 0 & 17 & 27 & 341 \\
\hline \multirow[t]{2}{*}{ Othet nontax revenues } & 52 & 8 & 124 & 142 & 203 \\
\hline & \multicolumn{5}{|c|}{ (In percent of total revenue) } \\
\hline Total revenne & 100.0 & 100.0 & 100.0 & 109.0 & 100.0 \\
\hline Tax revenue & 92.3 & 92.6 & 92.4 & 91.9 & 89.3 \\
\hline Taxes on income and profits & 19.2 & 18.1 & 14.0 & 13.5 & 15.6 \\
\hline Taxes an goods and services & 52.1 & 54.1 & 58.6 & 58.0 & 55.9 \\
\hline Taxes on intemational tade & 17.7 & 17.6 & 16.9 & 17.4 & 15.4 \\
\hline Other taxes & 3.4 & 2.8 & 2.9 & 3.0 & 2.4 \\
\hline Nomtax, revenus & 7.7 & 7.4 & 7.6 & 8.1 & 10.7 \\
\hline
\end{tabular}

Sources: Mozambican au thorities; und staff estimotes. 
Table 24. Mozambique: Locally Financed Public Investment by Sector, 1996-2000

\begin{tabular}{|c|c|c|c|c|c|}
\hline & 1996 & 1997 & 1998 & 1999 & 2000 \\
\hline & \multicolumn{5}{|c|}{ (In billions of meticais) } \\
\hline Agriculture and fishing & 22 & 39 & 34 & 70 & 152 \\
\hline Industry, energy, and mining & 6 & 18 & 25 & 37 & 49 \\
\hline Construction and water & 231 & 215 & 396 & 445 & 761 \\
\hline Transport and communications & 14 & 16 & 19 & 3 & 10 \\
\hline Education & 71 & 72 & 94 & 139 & 279 \\
\hline Health & 51 & 46 & 60 & 61 & 104 \\
\hline Government & 383 & 354 & 510 & 962 & 1,360 \\
\hline \multirow[t]{2}{*}{ Total } & 778 & 761 & 1,138 & 1,717 & 2,714 \\
\hline & \multicolumn{5}{|c|}{ (ln percent of total) } \\
\hline Agriculture and fishing & 2.8 & 5.2 & 3.0 & 4.1 & 5.6 \\
\hline Industry, energy, and mining & 0.8 & 2.4 & 2.2 & 2.2 & 1.8 \\
\hline Construction and water & 29.7 & 28.3 & 34.8 & 25.9 & 28.0 \\
\hline Transport and communications & 1.9 & 2.1 & 1.6 & 0.2 & 0.4 \\
\hline Education & 9.1 & 9.5 & 8.3 & 8.1 & 10.3 \\
\hline Health & 6.6 & 6.0 & 5.3 & 3.6 & 3.8 \\
\hline Government & 49.2 & 46.6 & 44.8 & 56.0 & 50.1 \\
\hline Total & 100.0 & 100.0 & 100.0 & 100.0 & 100.0 \\
\hline
\end{tabular}

Source: Mozambican authorities. 


$$
-31 \text { - }
$$

Táble 25. Mozambique: Monetary Survey, December 1997 - December 2001

(ln billions of meticais, wleşs ptherwise spocified)

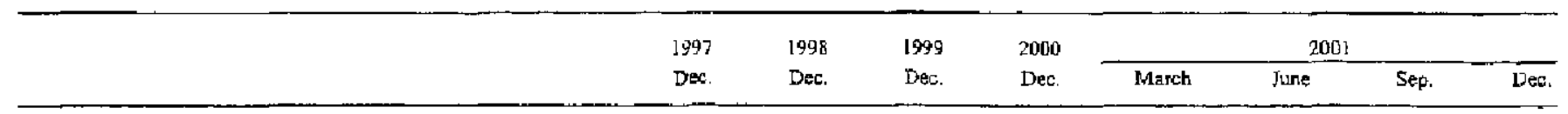

\section{Central bank}

Yier toreign assets

(in millions of U.S. dollars)

Net intethational reserves

(in millions of $\cup . S$. doilars)

Mraliums and long-tem fareign liabilities

Other

Net domestic assets

Credit to govemment (net)

Credit to banks (ne?)

Credit to the econarny

Qther items (net, assets +)

Reserve money

Currency outside barks

Bank teserves

Clerency in banks

Deposits in metistis

Required resaves (calculated)

Free reserves (calculated)

Deposits in foreign currencies

(in miltians of U.S. dollars)

$\begin{array}{rrrr}-2,653 & -1,973 & -1,384 & -99 \\ -230 & -160 & -104 & -6 \\ 4,013 & 5,214 & 6,242 & 9,015 \\ 344 & 422 & 469 & 526 \\ -6,830 & -7,338 & -7,819 & -4,357 \\ 160 & 151 & 197 & 243 \\ 5,444 & 4,665 & 4,501 & 4,039 \\ -3,852 & -4,898 & -5,175 & -5,433 \\ 567 & 454 & 408 & 483 \\ 55 & 75 & 74 & 1 \\ 8,675 & 9,031 & 9,194 & 8,989 \\ 2,792 & 2,692 & 3,117 & 3,940 \\ 1,544 & 1,650 & 2,174 & 2,425 \\ 1,248 & 1,042 & 943 & 1,515 \\ 282 & 269 & 391 & 428 \\ 361 & 639 & 237 & 845 \\ 473 & 530 & 710 & 1,246 \\ 388 & 109 & -473 & -401 \\ 105 & 134 & 315 & 242 \\ 9 & 11 & 24 & 14\end{array}$

$\begin{array}{rr}-258 & 1,451 \\ -14 & 66 \\ 9,677 & 11,517 \\ 518 & 525 \\ -10,200 & -10,390 \\ 265 & 324 \\ 3,985 & 3,246 \\ -6,055 & -5,645 \\ 674 & 1,604 \\ 1 & 1 \\ 9,366 & 7,286 \\ 3,729 & 4,697 \\ 2,419 & 2,621 \\ 1,309 & 2,076 \\ 321 & 343 \\ 813 & 1,614 \\ 1,298 & 1,456 \\ -485 & 158 \\ 175 & 119 \\ 9 & 5\end{array}$

$\begin{array}{rr}803 & 1,764 \\ 36 & 76 \\ 11,060 & 12,440 \\ 496 & 553 \\ -10,527 & -20,964 \\ 330 & 344 \\ 4,715 & 4,293 \\ -5,121 & 4,581 \\ 1,096 & 476 \\ 1 & 0 \\ 8,739 & 8,397 \\ 5,518 & 6,056 \\ 2,909 & 2,970 \\ 2,609 & 3,086 \\ 322 & 610 \\ 2,093 & 2,211 \\ 2,285 & 2,261 \\ -192 & -175 \\ 194 & 265 \\ 9 & 11\end{array}$

Deposit moncy banks (DMEBs)

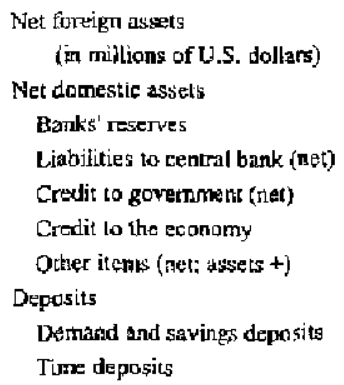

$\begin{array}{rrrr}1,784 & 1,721 & 2,109 & 4,449 \\ 155 & 139 & 159 & 260 \\ 4,086 & 5,350 & 7,502 & 9,904 \\ 1,390 & 1,310 & 1,013 & 1,545 \\ -555 & -337 & -397 & -959 \\ -576 & -714 & -441 & 296 \\ 5,350 & 6,649 & 8,645 & 11,343 \\ -1,523 & -1,550 & -1,4018 & -2,331 \\ 5,869 & 7,070 & 9,6] 1 & 14,353 \\ 4,579 & 5,370 & 7,293 & 10,775 \\ 1,290 & 1,701 & 2,318 & 3,579\end{array}$

3,985
213
11,232
1,679
-635
1,296
11,952
$-3,062$
15,218
11,358
3,859

5.815

265
11,111

2,245

$-1,901$

1,281

12,950

$-3,455$

16,926

12,090

4,835

$\begin{array}{rr}6,077 & 6,046 \\ 274 & 259 \\ 12,118 & 12,747 \\ 2,783 & 3,307 \\ -1,460 & -628 \\ 677 & 334 \\ 13,604 & 13,943 \\ -3,486 & -4,210 \\ 18,195 & 18,793 \\ 13,216 & 14,034 \\ 4,979 & 4,759\end{array}$

\section{Monetary survey}

Weat foreign assets

Net domestic assecs

Domastic credjt

Credit to government (net)

Credit to the ecomorny

Other items (net; asser + )

Of which: discrepancy in BoM'DMBs net claims

Money and quasi money (M2)

Curtency outside barlis

Deposics

Of which : foreign curtercy deposits

(i) milijons of U.S. dollars]
$-869$

8,282

977

$-4,429$

5,406

7,306

154

7,414

1,544

5,869

2,384
$-253$

8,973

$1,1 \$ 2$

$-5,612$

6,725

7,860

386

8,720

1,650

7.070

3,045
725

11,059

3,104

$-5,616$

8,720

7,956

170

11,785

2,174

9,61 !

4,151
4,350

12,429

6,207

$-5,137$

11,344

6,222

$-446$

16.779

2,425

14,353

7.ta?
3,727

13,908

7,295

$-4,757$

$1 !, 952$

6,713

408

17,637

2.,419

15,218

7,876

422
7,2606

12,281

8,587

4,364

12,951

3,694

$-128$

19,547

2,621

I6,926

9,36:
6,880

14,224

9,161

$-4,444$

13.605

5,063

$-191$

21,104

2,909

18,195

9,799
7,810

t3,954

9,697

4,246

13,944

4,256

21,763

2,970

18,793 9,897

Memorandurt items:

M2 growtl (12-snonth percent charge)

M2 growth at constant end-of-period exch. ratcs (in percent)

Credit to the economy (12-monib percent change)

Currency/M2 (in percent)

Velocity (GDP/average M2)

Market exchange rate (meticais per U.S. doltaj)

Source: Bank of Mozamoique (BoM).

\begin{tabular}{rrrrrrrr}
24 & 18 & 35 & 42 & 41 & 43 & 45 & 30 \\
24 & 15 & 32 & 29 & 24 & 25 & 26 & 12 \\
52 & 24 & 30 & 30 & 29 & 30 & 31 & 23 \\
21 & 19 & 18 & 14 & 14 & 13 & 14 & 14 \\
7 & 6 & 5 & 4 & $\ldots$ & $\ldots$ & $\ldots$ & 4 \\
11,547 & 12,366 & 19,300 & 17,131 & 18,667 & 21,933 & 22,182 & 23,320 \\
\hline
\end{tabular}


Table 26. Mozambique: Interest Rates, 1997-2001

(In percent per annum)

\begin{tabular}{|c|c|c|c|c|}
\hline & \multicolumn{2}{|c|}{ Commercial Banks 1/ } & \multirow{2}{*}{$\begin{array}{r}\text { Interbank Money } \\
\text { Market } 1 / \\
\end{array}$} & \multirow{2}{*}{$\begin{array}{l}\text { Central Bank } \\
\text { Rediscount } 2 / \\
\end{array}$} \\
\hline & Deposit & Lending & & \\
\hline \multicolumn{5}{|l|}{1997} \\
\hline Q1 & 30.10 & 35.80 & $\ldots$ & 24.75 \\
\hline $\mathrm{Q}^{2}$ & 25.00 & 32.30 & $\ldots$ & 23.50 \\
\hline Q3 & 14.90 & 33.80 & $\ldots$ & 19.95 \\
\hline Q4 & 11.10 & 27.50 & $\cdots$ & 12.95 \\
\hline \multicolumn{5}{|l|}{1998} \\
\hline$Q^{1}$ & 8.67 & 24.28 & $\cdots$ & 12.95 \\
\hline $\mathrm{Q}_{2}$ & 8.60 & 25.44 & $\ldots$ & 9.95 \\
\hline$Q 3$ & 7.76 & 24.70 & 10.04 & 9.95 \\
\hline Q4 & 7.86 & 22.99 & 10.24 & 9.95 \\
\hline \multicolumn{5}{|l|}{1999} \\
\hline Q1 & 7.86 & 19.63 & 9.76 & 9.95 \\
\hline Q2 & 7.86 & 19.63 & 9.51 & 9.95 \\
\hline Q3 & 7.86 & 19.63 & 9.77 & 9.95 \\
\hline $\mathrm{Q} 4$ & 7.86 & 19.63 & 10.64 & 9.95 \\
\hline \multicolumn{5}{|l|}{2000} \\
\hline Q1 & 8.75 & 19.41 & 10.74 & 9.95 \\
\hline Q2 & 9.56 & 18.73 & 15.17 & 9,95 \\
\hline Q3 & 9.79 & 18.44 & 17.71 & 9.95 \\
\hline Q4 & 10.68 & 19.58 & 20.88 & 9.95 \\
\hline \multicolumn{5}{|l|}{2001} \\
\hline Q1 & 13.36 & 20.50 & 31.63 & 9.95 \\
\hline $\mathrm{Q}^{2}$ & 13.75 & 21.20 & 30.89 & 9.95 \\
\hline $\mathrm{Q}^{3}$ & 15.44 & 23.87 & 31.59 & 9.95 \\
\hline Q4 & 17.86 & 26.04 & 38.50 & 9.95 \\
\hline
\end{tabular}

Source: Bank of Mozambique.

1/ Quarterly arithmetic average of rates offered by banks.

2/ End of quarter. 
Table 27. Mozambique: Balance of Payments, 1997-2001

(In millions of U.S. dollarg, unless otherwise specified)

\begin{tabular}{|c|c|c|c|c|c|}
\hline & 1997 & 1998 & 1999 & 2000 & 2001 \\
\hline Ttade balatice & -530.0 & -572.7 & -916.1 & -798.3 & -413.9 \\
\hline Exparts, folb. & 230.0 & 244.6 & 283.7 & 364,0 & 703.6 \\
\hline Imports, $\mathbf{c}_{\mathrm{i}} \mathrm{i}, \mathrm{f}$. & .760 .0 & -817.3 & $-1,199.8$ & $-1,162.3$ & $-1,117.5$ \\
\hline Services (net) & -80.8 & -176.3 & -236.0 & -243.3 & -435.7 \\
\hline Receipts & 342.3 & 332.5 & 355,6 & 405.1 & 347,9 \\
\hline Expenditures & -423.1 & -508.8 & .5916 & -648.4 & -783.6 \\
\hline Of which: interest on public debt & -140.2 & 150.2 & -161.6 & -160.8 & -146.8 \\
\hline Cursent account, excluding grants & 0.0 & 0.0 & $-1,152.1$ & $-1,041.6$ & -349.5 \\
\hline Cinreguited official transfors & 312.9 & 313.2 & 434.1 & 563.9 & 469.3 \\
\hline Curtent account, inciuding grants & 312.9 & 313.2 & -718.0 & -477.7 & -380.2 \\
\hline Capital account & 180.8 & 262.8 & 613.4 & 278,9 & -0.1 \\
\hline Foretgn berrowing & 315.3 & 299.5 & 472.0 & 483.8 & 209.7 \\
\hline Public & 226.3 & 218.1 & 111.7 & 161.7 & 1039 \\
\hline Private 1/ & 90.0 & 81.4 & 360.3 & 322.1 & 105.8 \\
\hline Anorization. & -201.0 & -249.4 & .240 .3 & -344.0 & -392.3 \\
\hline Public & -180.0 & -211.2 & -200.7 & -305.5 & -3059 \\
\hline Priwate $/ /$ & -21.0 & -38.3 & -39.6 & -37.5 & -86.4 \\
\hline Direct investment (net) & 64.4 & 212.7 & 381.7 & 139.1 & 182.5 \\
\hline Short-term capital and entors and onissions (net) & 19.3 & -31.4 & -131.3 & -152.5 & -38.4 \\
\hline Of which : comtmercial banks" net foreign asset (increase -) & 33.5 & 15.3 & $-20,2$ & -95.7 & 0.0 \\
\hline Overall balance & -97.6 & -204.4 & -235.9 & -351.3 & -418.7 \\
\hline Financing & 97.6 & -56.8 & -808.4 & -980 & -6.7 \\
\hline Bank of Mozambique net forejgon asset (increase -) & 0.0 & 0.0 & -46.9 & .98 .0 & -6.7 \\
\hline Gross international reserves (increase-) 3 . & -167.7 & -92.9 & -44.1 & -129.1 & 10.1 \\
\hline Use of INF credit (net) & 19.6 & 9.6 & -2.8 & 31.1 & -16.8 \\
\hline Other (Jet) & 0.0 & 6.1 & 0.0 & 0.0 & 0.0 \\
\hline Net change in artears (jncrease $\rightarrow$ ) & -3932.2 & 20.4 & $-76 ! .5$ & 0.0 & 0.0 \\
\hline Financing gap before debt tclief & 0.0 & 261.2 & 1044.3 & 449,3 & 425.4 \\
\hline Financing gap after debt telief & 0.0 & 0.0 & 0.0 & 0.0 & -0.1 \\
\hline \multicolumn{6}{|l|}{ Mentorandum items: } \\
\hline Debt relief 2 & 4177.9 & 261.2 & 10443 & 449.3 & 425.5 \\
\hline \multicolumn{6}{|l|}{ Curtent account deficit (in pencent of GDP) } \\
\hline Excluding grants & 17.7 & 18.9 & 28.2 & 26.8 & 23.6 \\
\hline Jncluding granis & 8.6 & 11.0 & 17.5 & 11.8 & 10.5 \\
\hline Gross interational reserves & 532.0 & 624.9 & 669.3 & 745.8 & 727.0 \\
\hline (in months of imports of goods and nanfactor services) & 6.8 & 7.8 & $\$ .5$ & 6]$. & 6.4 \\
\hline \multicolumn{6}{|l|}{ Debt indicators } \\
\hline Net prescrt value of DebuGDP & $\cdots$ & ... & 19.8 & 24.6 & 25.3 \\
\hline Net present value of Debt/exports & $\ldots$ & $\ldots$ & 212.0 & 194.4 & 116.1 \\
\hline Debt service due/exports & $\cdots$ & $\ldots$ & 15.3 & 9.1 & 3.5 \\
\hline Debt service due/reventus & $\ldots$ & $\ldots$ & 17.5 & 9.4 & 5.8 \\
\hline
\end{tabular}

Sources: Mozambican authorities; and staff estimates.

If Private botrowing, ngt guaranteed by the govemment or the Bank of Mozambique.

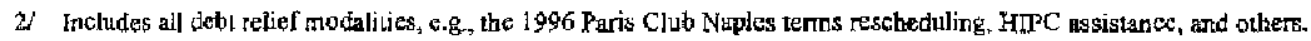


Table 28. Mozambique: Foreign Trade Indicators, 1997-2001 1/

\begin{tabular}{|c|c|c|c|c|c|}
\hline & 1997 & 1998 & 1999 & 2000 & 2001 \\
\hline & \multicolumn{5}{|c|}{ (Indices, $1990=100)$} \\
\hline \multicolumn{6}{|l|}{ Exports } \\
\hline Value & 182.0 & 166.3 & 164.5 & 187.4 & 208.0 \\
\hline Prices 21 & 93.4 & 84.0 & 71.8 & 71.4 & 71.9 \\
\hline Volume $3 /$ & 194.9 & 197.9 & 229.1 & 262.6 & 289.0 \\
\hline \multicolumn{6}{|l|}{ Inports } \\
\hline Value & 97.4 & 99.1 & 93.0 & 141.7 & 124.6 \\
\hline Prices $4 /$ & 97.4 & 90.7 & 90.4 & 92.5 & 88.1 \\
\hline Volume $3 /$ & 100.0 & 109.2 & 102.9 & 153.3 & 141.3 \\
\hline Terms of trade & 95.8 & 92.6 & 79.4 & 77.2 & 81.6 \\
\hline & \multicolumn{5}{|c|}{ (Annual percentage changes) } \\
\hline \multicolumn{6}{|l|}{ Exports } \\
\hline Value & 1.7 & -8.7 & -1.0 & 13.9 & 11.0 \\
\hline Prices $2 /$ & 1.4 & -10.0 & -14.5 & -0.6 & 0.8 \\
\hline Volume 3/ & 0.3 & 1.5 & 15.8 & 14.6 & 10.1 \\
\hline \multicolumn{6}{|l|}{ Imports } \\
\hline Value & -2.9 & 1.7 & -6.1 & 52.4 & -12.1 \\
\hline Prices 4 & -7.1 & -6.9 & -0.3 & 2.2 & -4.7 \\
\hline Volume $3 /$ & 4.6 & 9.3 & -5.8 & 49.0 & -7.8 \\
\hline Terms of trade & 9.2 & -3.3 & -14.3 & -2.8 & 5.8 \\
\hline
\end{tabular}

Source: Staff estimates, based on data from the Bank of Mozambique and IMF, International Financial Statistics.

1/ Values and prices in U.S. dollar terms.

2) Weighted average of unit values with constant weights according to the share of each item in the total of 1999.

3/ Implicit volune derived from value and prices.

4/ From international price indices (e.g., cost and prices of partner suppliers) and estimates of the Research Department, IMF. 
Table 29. Mozambique: Commodity Composition of Exports, 1997-2001 1/

(Value in millions of U.S. dollars; volume in thousands of metric tons; and unit value in U.S. dollars per metric ton)

\begin{tabular}{|c|c|c|c|c|c|}
\hline & 1997 & 1998 & 1999 & 2000 & 20012 \\
\hline Total exports, f.o.b. & 230.0 & 244.6 & 283.7 & 364.0 & 703.6 \\
\hline Aluminum $3 /$ & 0.0 & 0.0 & 0.0 & 60.2 & 92.7 \\
\hline Prawns, value & 85.1 & 72.6 & 65.5 & 91.5 & 92,4 \\
\hline Volume & 11.1 & 9.8 & 8.6 & $\ldots$ & ... \\
\hline Unit value & $7,668.6$ & $7,408.9$ & $7,631.2$ & $\ldots$ & ... \\
\hline Electricity & 0.0 & 36.2 & 62.9 & 67.0 & 57.3 \\
\hline Cotton, value & 25.2 & 22.3 & 19.9 & 25.5 & 18.3 \\
\hline Volume & 15.6 & {$[8.7$} & 20.5 & $\ldots$ & $\ldots$ \\
\hline Unit value & $1,611.3$ & $1,194,6$ & 968.8 & $\cdots$ & ... \\
\hline Manufacturing products & $19: 9$ & 14.3 & 13.9 & $\ldots$ & 17.3 \\
\hline Timber & 13.8 & 11.0 & 8.8 & 14.2 & 12.6 \\
\hline Processed cashew nuts, value & 0.0 & 19.1 & 7.8 & 8.4 & 10.9 \\
\hline Volume & 3.8 & 4.9 & 1.9 & $\cdots$ & $\cdots$ \\
\hline Unit value & $3,713.1$ & $3,894.2$ & $4,175.8$ & $\cdots$ & $\cdots$ \\
\hline Unprocessed cashew nuts & 15.1 & 21.6 & 25.1 & 11.9 & 10.9 \\
\hline Petroleum, value 4/ & $2: 4$ & 1.3 & 4.4 & 9.0 & 9.6 \\
\hline Sugar, value & 12.8 & 8.4 & 5.3 & 4.3 & 8.0 \\
\hline Volume & 31.9 & 20.4 & 13.9 & $\ldots$ & $\ldots$ \\
\hline Unit value & 402.3 & 413.3 & 395.9 & $\ldots$ & $\cdots$ \\
\hline Tires and tubes & 3.3 & 3.4 & 1.0 & 3.6 & 4.6 \\
\hline Copra, value & 4.6 & 5.0 & 3.5 & 2.1 & 0.8 \\
\hline Volume & 14.1 & 18.2 & 16.6 & & \\
\hline Unit value & 326.4 & 276.0 & 210.8 & $\cdots$ & $\cdots$ \\
\hline Citrus, value & 0.8 & 0.4 & 5.8 & 0.8 & 0.3 \\
\hline Volume & 2.6 & 3.6 & 57.8 & $\ldots$ & ... \\
\hline Unit value & 286.8 & 101.5 & 100.3 & $\cdots$ & $\cdots$ \\
\hline Fishery products $5 /$ & 7.2 & 5.6 & 8.9 & $\cdots$ & $\ldots$ \\
\hline Other & 39.9 & 23.3 & 50.9 & 65.5 & 385.3 \\
\hline
\end{tabular}

Sources: Mozambican authorities; and staff estimates.

1/ Official statistics for the years 2000 and 2001 do not provide for information on export volumes and prices.

2/ Based on preliminary data,

3/ The data for aluminum exporls in 2001 are freliminary and underestimate actual aluminum exports; according to the authorities, a latge part of alaminum exports ase included under the item Other for the year 2001. Also refer to footnote 2 of Table 30.

4/ Petroleurn products, including bunkers.

5/ Excludes prawns. 
Table 30. Mozambique: Exports by Country of Destination, 1997-2001

(In percent of total exports, unless otherwise indicated)

\begin{tabular}{|c|c|c|c|c|c|}
\hline & 1997 & 1998 & 1999 & 2000 & $2001 \mathrm{~V}$ \\
\hline OECD countries & 57.3 & 37.8 & 37.3 & 39.6 & 14.8 \\
\hline Japan & 8.4 & 4.8 & 4.3 & 4.3 & 4.2 \\
\hline Netheriands & 3.0 & 2.3 & 1.7 & 1.0 & 1.0 \\
\hline Portugal & 8.9 & 7.6 & 9.0 & 11,6 & 4.0 \\
\hline Spain & 18.5 & 13.0 & 12.7 & 10.7 & 3.8 \\
\hline United Kingdom & 0.7 & 1.4 & 1.0 & 0.9 & 0.0 \\
\hline United States & 11.5 & 5.7 & 4.7 & 4.7 & 0.9 \\
\hline Other & 6.3 & 3.0 & 3.9 & 6.4 & 0.8 \\
\hline Other countries & 42.7 & 62.2 & 62.7 & 60,4 & 85.2 \\
\hline South Africa & 17.5 & 17.6 & 26.2 & 14.6 & $1 \$ 3$ \\
\hline Zinbabwe & 4.1 & 19.5 & 14.9 & 17.7 & 5.3 \\
\hline Other $2 l$ & 21.1 & 25.1 & 21.7 & 28.1 & 64.6 \\
\hline Total & 100.0 & 100.0 & 100.0 & 100.0 & 100.0 \\
\hline \multicolumn{6}{|l|}{ Mernorandum item: } \\
\hline Total exports (millions of U.S. dollars) & 230.0 & 244.5 & 283.7 & 364.0 & 703.6 \\
\hline
\end{tabular}

Source: Mozambican authorities; and IMF, Direction of Trade Statistics.

1/ Based on preliminary data.

2) Data for 2001 include exports from Mozambique Aluminum (MOZAL) to the European Unios (EU), almost $85 \%$ of the total; the statistics do not provide for a disaggregate view per EU member country. 
Table 31. Mozambique: Imports by Country of Origin, 1997-2001

(In percent of total imports, unless otherwise indicated)

\begin{tabular}{|c|c|c|c|c|c|}
\hline & 1997 & 1998 & 1999 & 2000 & 20011 \\
\hline OECD countries & 37.3 & 31.9 & 27.2 & 28.6 & 21.9 \\
\hline Erance & 2.7 & 2.1 & 0.9 & 2.2 & 1.1 \\
\hline Japan & 4.5 & 3.9 & 3.9 & 4.5 & 0.6 \\
\hline Netherlands & 1.3 & 0.8 & 0.7 & 0.5 & 0.9 \\
\hline Portugal & 6.1 & 7.9 & 5.6 & 7.6 & 8.4 \\
\hline United Kingdom & 2.1 & 2.0 & 0.9 & 1.6 & 1.1 \\
\hline United States & 5.2 & 5.3 & 6.7 & 3.5 & 1.8 \\
\hline Other & 15.4 & 9.9 & 8.5 & 8.6 & 7.9 \\
\hline Other countries & 62.7 & 68.1 & 72.8 & 71.4 & 78.1 \\
\hline South Africa & 40.9 & 39.5 & 57.2 & 49.8 & 40.5 \\
\hline Zimbabwe & 2.3 & 2.3 & 0.5 & 0.6 & 0.8 \\
\hline Other $2 /$ & 19.5 & 26.3 & 15.1 & 21.0 & 36.8 \\
\hline Total & 100.0 & 100.0 & 100.0 & 100.0 & 100.0 \\
\hline Memorandum ftem: & & & & & \\
\hline Total imports (millions of U.S. dollars) & -760.0 & -817.3 & $-1,199.8$ & $-1,162.3$ & $-1,117.5$ \\
\hline
\end{tabular}

Source: Mozambicatt authorities; and IMF, Direction of Trade Statistics.

1/ Based on preliminary data.

2/ About half of the import data for 2001 originate in the European Union. The statistic does not allow for country specific disaggregation. 
Table 32. Mozambique: Exchange Rates, 1980-2002

\begin{tabular}{|c|c|c|c|c|c|c|}
\hline \multicolumn{2}{|c|}{$\begin{array}{l}\text { Effective exchange rates } \\
\text { (Index, 1990=100) }\end{array}$} & \multicolumn{2}{|c|}{$\begin{array}{c}\text { Official } \\
\text { meticais pex U.S. dollar lit }\end{array}$} & \multicolumn{2}{|c|}{$\begin{array}{c}\text { Market } \\
\text { melicais per U.S. dollar U/ }\end{array}$} & \multirow{2}{*}{$\begin{array}{l}\text { Cashiparalled tharket } \\
\text { meticais per U.S. doltar 1/2/ } \\
\text { Maputo } \\
\text { average }\end{array}$} \\
\hline Real & Nominal & $\begin{array}{l}\text { End of } \\
\text { period }\end{array}$ & $\begin{array}{l}\text { Period } \\
\text { average }\end{array}$ & $\begin{array}{l}\text { End of } \\
\text { period }\end{array}$ & $\begin{array}{l}\text { Period } \\
\text { average }\end{array}$ & \\
\hline
\end{tabular}

Annual

\begin{tabular}{|c|c|c|c|c|c|c|c|}
\hline 1980 & 117.6 & $1,572.8$ & 33 & 32 & $\ldots$ & $\ldots$ & $\ldots$ \\
\hline 1981 & 115.3 & $1,663.3$ & 36 & 35 & $\ldots$ & $\ldots$ & $\ldots$ \\
\hline 1982 & 134.2 & $1,822.9$ & 38 & 38 & $\ldots$ & $\ldots$ & $\cdots$ \\
\hline 1983 & 163.4 & $1,880.4$ & 41 & 40 & $\ldots$ & $\ldots$ & $\ldots$ \\
\hline 1984 & 220.2 & $2,099.7$ & 44 & 42 & $\ldots$ & $\ldots$ & $\ldots$ \\
\hline 1985 & 306.3 & $2,431.2$ & 41 & 43 & $\ldots$ & $\ldots$ & $\ldots$ \\
\hline 1986 & 377.4 & $2,305.3$ & 39 & 40 & $\ldots$ & $\ldots$ & $\ldots$ \\
\hline 1987 & 147.7 & 437.3 & 404 & 292 & $\ldots$ & $\cdots$ & 1,096 \\
\hline 1988 & 95.6 & 160.3 & 626 & 529 & $\cdots$ & $\ldots$ & 1,183 \\
\hline 1989 & 99.2 & 125.8 & 820 & 745 & $\ldots$ & $\ldots$ & 1,933 \\
\hline 1990 & 100.0 & 100.0 & 1,038 & 929 & 1,879 & $\ldots$ & 2,155 \\
\hline 1991 & 84.7 & 72.0 & 1,845 & 1,434 & 2,033 & 1,957 & 2,194 \\
\hline 1992 & 65.3 & 43.1 & 3,742 & 2,433 & 2,951 & 2,628 & 2,894 \\
\hline 1993 & 64.6 & 34.3 & 5,238 & 3,723 & 5,343 & 4,081 & 4,500 \\
\hline 1994 & 62.8 & 23.5 & 6,553 & 5,918 & 6,651 & 6,152 & 6,679 \\
\hline 1995 & 59.9 & 15.6 & 10,776 & 8,690 & 10,890 & 9,022 & $9,61 \mathrm{I}$ \\
\hline 1996 & 68.3 & 12.5 & 11.295 & 11,140 & $11_{5} 377$ & 11,294 & 11,909 \\
\hline 1997 & 74.0 & 13.2 & 31,604 & 11,395 & 11,543 & 11,546 & 11,708 \\
\hline 1998 & 76.6 & 14.0 & 12,366 & $1], 8 \subseteq 3$ & {$[2,366$} & 11,850 & 12,057 \\
\hline 1999 & 73.9 & 13.6 & 13,238 & 12,673 & {$[3,300$} & 12,689 & 12,844 \\
\hline 2000 & 72.8 & 12.3 & 16,985 & 15,341 & 17,140 & 15,689 & 15,996 \\
\hline 2001 & 61.7 & 9.9 & 23,320 & 20,707 & 23,320 & 20,707 & 21,139 \\
\hline
\end{tabular}

Quarterly

\begin{tabular}{|c|c|c|c|c|c|c|c|c|}
\hline \multirow{4}{*}{1927} & Q1 & 71.1 & 12.8 & 11,400 & 11,354 & I 1,493 & 11.512 & 11,733 \\
\hline & $\mathrm{Q} 2$ & $72 . !$ & 13.0 & $11,4 \geq 1$ & 11,382 & 11,395 & 11,529 & 11,700 \\
\hline & $Q^{3}$ & 75.9 & 13.4 & 11,396 & 11,388 & 11,655 & 11,561 & 11,700 \\
\hline & $Q 4$ & 76.7 & 13.6 & 11,604 & 11,454 & 11,343 & 11,580 & 11,700 \\
\hline \multirow{4}{*}{1998} & Q! & 78.6 & 14.2 & 11,625 & $11,6.38$ & 11,625 & 11,638 & 11,833 \\
\hline & $Q 2$ & 78.0 & 14.2 & 11,813 & 11,647 & 11,813 & 11,665 & 11,920 \\
\hline & $Q 3$ & 76.9 & 14.2 & 12,044 & 11,933 & 12,074 & 11,923 & 12,079 \\
\hline & $Q_{4}$ & 72.9 & 13.5 & 12,366 & 17,196 & 12,366 & 12,176 & 12,393 \\
\hline \multirow[t]{4}{*}{1999} & $\mathrm{Q}^{1}$ & 74.5 & 13.7 & 12,450 & 12,413 & 12,438 & 12,388 & 12,533 \\
\hline & $\mathrm{Q} 2$ & 75.8 & 13.9 & 12,604 & 12,525 & 12,546 & 12,499 & 12,747 \\
\hline & $\mathrm{Q}^{3}$ & 73.2 & 13.4 & $12,8 \mathrm{~B} 6$ & 12,710 & 12,933 & 12,764 & 12,823 \\
\hline & Q4 & 72.1 & 13.3 & $13,21 \mathrm{~B}$ & 13,045 & 13,300 & 13,106 & 13,274 \\
\hline \multirow[t]{4}{*}{2000} & Q1 & 73.2 & 12.9 & 14,513 & 13,765 & 15,497 & 14,121 & $14,39]$ \\
\hline & $\mathrm{Q} 2$ & 72.3 & 120 & 15,273 & 15,032 & 16,070 & 15,926 & 16,256 \\
\hline & $Q: 3$ & 73.1 & 12.0 & 15,592 & 15,426 & 16,139 & 16,130 & 16,446 \\
\hline & $Q_{4}$ & 72.6 & 12.0 & 16,985 & 16,341 & 17,140 & 16,581 & 16,890 \\
\hline \multirow[t]{4}{*}{2001} & $\mathrm{Q} 1$ & 63.1 & 20.9 & 18,575 & 17,783 & 18,667 & 17,895 & 18,356 \\
\hline & $Q 2$ & 61.2 & 10.3 & 21,560 & 19,911 & 21,933 & 20,162 & 20,675 \\
\hline & $Q 3$ & 59.6 & 9.3 & 22,182 & 21,895 & 22,182 & 21,895 & 22,247 \\
\hline & $Q^{4}$ & 63.2 & 9.0 & 23,320 & 22,877 & 23,320 & 22,877 & 23,278 \\
\hline 2002 & Q1 & 62.7 & 9.0 & 23,854 & 23,447 & 23,854 & 23,447 & $\ldots$ \\
\hline
\end{tabular}

Sources: Bank of Mozambique; and IMF, Mntamational Financial Statssics.

$1 /$ Midpoint official exchange rates; buying and selling rates plysiminus I percent.

$2^{\prime}$ A more depreciated rate for the thetical is usually offered on cash denominacions transacted without record by infomal operawors and some foreign exchange bureaus. 